\title{
UNE APPROCHE GÉOMÉTRIQUE DU CONTRÔLE OPTIMAL DE L'ARC ATMOSPHÉRIQUE DE LA NAVETTE SPATIALE
}

\author{
BERNARD BONNARD ${ }^{1}$ ET EMMANUEL TRÉLAT ${ }^{2}$
}

\begin{abstract}
The aim of this article is to make some geometric remarks and some preliminary calculations in order to construct the optimal atmospheric arc of a spatial shuttle (problem of reentry on Earth or Mars Sample Return project). The system describing the trajectories is in dimension 6, the control is the bank angle and the cost is the total thermal flux. Moreover there are state constraints (thermal flux, normal acceleration and dynamic pressure). Our study is mainly geometric and is founded on the evaluation of the accessibility set taking into account the state constraints. We make an analysis of the extremals of the Minimum Principle in the non-constrained case, and give a version of the Minimum Principle adapted to deal with the state constraints.
\end{abstract}

Résumé. L'objectif de ce travail est de faire quelques remarques géométriques et des calculs préliminaires pour construire l'arc atmosphérique optimal d'une navette spatiale (problème de rentrée sur Terre ou programme d'exploration de Mars). Le système décrivant les trajectoires est de dimension 6 , le contrôle est l'angle de gîte cinématique et le coût est l'intégrale du flux thermique. Par ailleurs il y a des contraintes sur l'état (flux thermique, accélération normale et pression dynamique). Notre étude est essentiellement géométrique et fondée sur une évaluation de l'ensemble des états accessibles en tenant compte des contraintes sur l'état. On esquisse une analyse des extrémales du Principe du Minimum dans le cas non contraint et l'on cite un Principe du Minimum adapté à analyser le problème avec contraintes sur l'état.

Classification Mathématique. 49K15, 70Q05.

Reçu le 31 mars 2001.

\section{INTRODUCTION}

L'objectif de ce travail est de faire une analyse préliminaire du problème de contrôle optimal de l'arc atmosphérique de la navette spatiale où le coût est l'intégrale du flux thermique qui est un facteur d'usure de la navette. Le système est mono-entrée et le contrôle est la gîte cinématique (l'angle d'attaque est fixé). Il y a des contraintes sur l'état : contraintes sur le flux thermique, l'accélération normale et la pression dynamique. Ce travail complète les deux travaux suivants. Le premier [3] est une approche purement numérique d'un problème

Mots-clés et phrases : Contrôle optimal avec contraintes sur l'état, principes du minimum, mécanique céleste, arc atmosphérique.

1 Université de Bourgogne, LAAO, Dijon, France ; e-mail : bbonnard@u-bourgogne.fr

2 Université Paris Sud, Orsay, France ; e-mail : trelat@topolog.u-bourgogne.fr 
de programmation non linéaire du problème discrétisé ; le problème est aussi simplifié par le fait que les auteurs n'imposent qu'une condition terminale sur le module du vecteur vitesse. Les résultats sont cependant intéressants car ils montrent que les contraintes sur l'état sont généralement saturées. Le second travail [9] est plus complet et s'appuie sur la méthode de Graves, Harpold pour le guidage de la navette américaine. L'approche est de planifier une trajectoire saturant les contraintes et de la stabiliser. Ce travail met bien en évidence le problème d'accessibilité car le système est mono-entrée et le contrôle est borné. L'article reflète aussi le problème crucial du choix des coordonnées pour analyser le transfert.

Notre approche est géométrique et utilise une évaluation de la frontière de l'ensemble des états accessibles paramétrisée par les conditions nécessaires d'optimalité dans l'esprit des travaux de [19,20] et [13], en prenant en compte les contraintes sur le contrôle et sur l'état. Les calculs utilisent des coordonnées spéciales fondées sur la structure de l'algèbre de Lie associée au système et la géométrie des contraintes. Cette approche est illustrée par l'exemple trivial suivant. Soit le problème du temps minimal pour le système lisse : $\dot{q}=X(q)+u Y(q)$ où $q \in \mathbb{R}$ et $|u| \leq 1$. Introduisons le système augmenté de $\mathbb{R}^{2}: \dot{q}=X(q)+u Y(q), \dot{t}=1$. Soit $q_{0}$ un point tel que le convexe $C\left(q_{0}\right)=\left\{X\left(q_{0}\right)+u Y\left(q_{0}\right),|u| \leq 1\right\}$ ne contienne pas 0 , sans nuire à la généralité on peut supposer que $\dot{q}>0$ en $q_{0}$. Représentons sur la figure 1 un ensemble d'états accessibles générique pour le système augmenté.

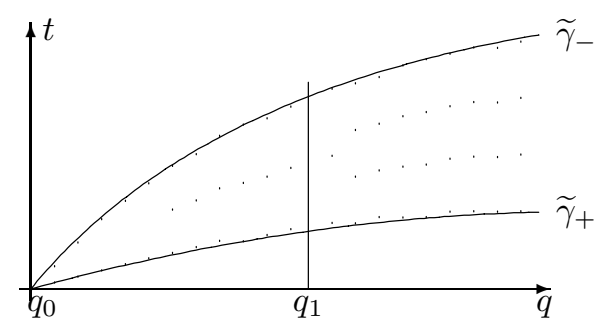

FIGURE 1

Sa frontière est formée par les deux trajectoires $\widetilde{\gamma}_{-}, \widetilde{\gamma}_{+}$correspondant respectivement à $u=-1$ et $u=+1$, et dans le cas de la figure 1 la politique $u=+1$ (resp. $u=-1$ ) est temps minimale (resp. maximale) pour joindre $q_{0}$ à $q_{1}$. L'analyse est globale tant que les courbes $\widetilde{\gamma}_{-}$et $\widetilde{\gamma}_{+}$ne sont pas tangentes. Aux points de tangence il faut affiner l'analyse. L'exemple suivant est moins trivial et prend en compte les contraintes.

Considérons le problème du temps minimal pour le système : $\dot{q}=X(q)+u Y(q),|u| \leq 1$. Soit $\gamma_{+}$(resp. $\gamma_{-}$) un arc correspondant à $u=+1$ (resp. $u=-1$ ) et notons $\gamma_{1} \gamma_{2}$ un arc $\gamma_{1}$ suivi par $\gamma_{2}$. Soit $q_{0}$ un point générique. L'ensemble des états accessibles en temps petit à partir de $q_{0}$ a la forme d'un cône bordé par $\gamma_{+}$ et $\gamma_{-}$, et chaque trajectoire optimale est de la forme $\gamma_{+} \gamma_{-}$ou $\gamma_{-} \gamma_{+}$, voir figure $2 \mathrm{i}$, et le temps le long d'une trajectoire est mesurable à l'aide de la forme de Miele : $\omega=p d q$, où $p$ vérifie $\langle p, X\rangle=1$ et $\langle p, Y\rangle=0$. Supposons que $q_{0}=0$ et que les trajectoires sont contraintes au domaine $C: y \geq 0$. Soit $\gamma_{b}(t), t \in[0, T]$ un arc frontière issu de 0 contenu dans la contrainte : $y=0$. Supposons que le contrôle associé est admissible et non saturant. Soit $B=\gamma_{b}(T), T$ assez petit. Parmi les arcs $\gamma_{+} \gamma_{-}$et $\gamma_{-} \gamma_{+}$joignant 0 à $B$, l'un est temps minimal et l'autre est temps maximal, pour le problème sans contraintes, voir figure 2ii. Supposons que ce soit $\gamma_{+} \gamma_{-}$. Alors s'il satisfait $y \geq 0$, il est admissible pour le problème contraint et l'arc frontière n'est pas optimal. De plus la synthèse optimale au voisinage de 0 est $\gamma_{+} \gamma_{-}$. Si $\gamma_{+} \gamma_{-}$n'est pas contenu dans $y \geq 0$, l'arc frontière est temps optimal et la synthèse optimale est $\gamma_{+} \gamma_{b} \gamma_{-}$. Pour faire les calculs en détails on peut utiliser le modèle : $\dot{x}=1+a y, \dot{y}=b+u$ et non la forme horloge de Miele, qui n'est valide que dans le cas du plan.

Dans les calculs de [19] le Principe du Maximum est une condition nécessaire que doivent vérifier les trajectoires frontières, si l'on relaxe la condition $p^{0} \leq 0$ où $p^{0}$ est la variable duale du temps, car la frontière contient les maxima et les minima, voir figure 1.

Un problème majeur pour analyser un problème optimal avec des contraintes est d'obtenir des conditions nécessaires d'optimalité. En effet les contraintes peuvent être pénalisées dans le coût de différentes façons. Cela conduit à définir le concept d'ordre des contraintes. C'est aussi le concept géométrique de base pour construire 


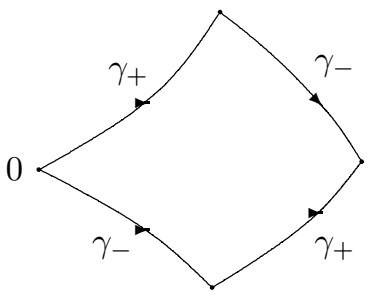

(i)

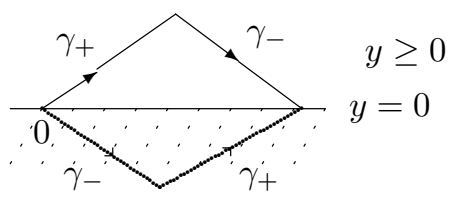

(ii)

FIGURE 2

des formes normales et évaluer l'ensemble des états accessibles pour le système avec contraintes. On formule un Principe du Minimum dû à [12] et [15] prenant en compte ce concept et qui est adapté pour analyser le problème de contrôle optimal de la navette. Il concerne les systèmes mono-entrée et exige des conditions de régularité. Il est bien plus précis que le Principe du Minimum général de [11] où une trajectoire optimale est la projection d'une trajectoire dans l'espace cotangent qui dépend d'une mesure supportée par les contraintes.

Enfin on doit préciser que notre approche n'est pas entièrement intrinsèque car on utilise la réduction de [9] identifiant la cible au pôle nord.

L'organisation de cet article est la suivante :

en section 2 on rappelle le modèle ;

en section 3 on présente le problème de contrôle optimal.

L'objectif de la section 4 est d'analyser les trajectoires extrémales pour le système non contraint. La structure des équations permet de distinguer un sous-système I, en dimension 3, qui correspond au problème de contrôle sans conditions limites sur la latitude et l'azimut. En utilisant la structure de l'algèbre de Lie du système, on décrit l'ensemble des états accessibles et la politique optimale en temps petit. On calcule ensuite pour le système complet les extrémales singulières et on caractérise leur statut d'optimalité. Enfin on initialise la classification des extrémales régulières pour le problème où le domaine de contrôle est convexifié.

Dans la section 5 on étudie le problème de contrôle optimal avec contraintes sur l'état. On rappelle un principe du minimum de [15] adapté à l'analyse du problème optimal pour la navette. On introduit la méthodologie nécessaire pour classifier les synthèses optimales au voisinage de la contrainte et on présente des résultats préliminaires pour des systèmes en dimension 2 et 3 . Ce travail est appliqué au sous-système I de la navette pour calculer la synthèse optimale au voisinage de la contrainte.

Nos calculs explicites concernent les temps petits et l'objectif de la section 6 est d'initialiser les simulations numériques pour le problème de rentrée atmosphérique, fondées sur le principe du minimum avec contraintes de $[15]$.

\section{LE MODÈLE}

Le problème est de contrôler l'arc atmosphérique au voisinage d'une planète qui peut être la Terre pour le problème de rentrée, ou Mars pour le programme d'exploration. Dans les deux cas les équations sont les mêmes sauf pour les paramètres spécifiques à chaque planète (rayon, masse, vitesse de rotation, densité de l'atmosphère, etc.). Dans nos calculs on va supposer que la planète est la Terre. Pour modéliser le problème, on utilise les lois de la mécanique classique, un modèle de densité atmosphérique et un modèle pour les forces s'exerçant sur la navette, la force gravitationnelle et la force aérodynamique qui se décompose en une composante dite de traînée et une composante dite de portance.

Les équations sont simplifiées en choisissant des repères mobiles adaptés. Par ailleurs en l'absence d'atmosphère la trajectoire de la navette est Keplerienne (si on néglige la rotation de la planète), et le système possède les 
intégrales premières d'un mouvement dans un champ central et une intégrale première dite de Laplace due à la nature du potentiel.

\subsection{Les repères mobiles}

On donne un modèle général tenant compte de la rotation (uniforme) de la Terre autour le l'axe $K=N S$, à vitesse angulaire de module $\Omega$. On note $E=\left(e_{1}, e_{2}, e_{3}\right)$ un repère galiléen dont l'origine est le centre $O$ de la Terre, $R_{1}=(I, J, K)$ un repère d'origine $O$ en rotation à la vitesse $\Omega$ autour de l'axe $K$, et $I$ l'intersection avec le méridien de Greenwich.

Soit $R$ le rayon de la Terre et $G$ le centre de masse de la navette. On note $R_{1}^{\prime}=\left(e_{r}, e_{l}, e_{L}\right)$ le repère associé aux coordonnées sphériques de $G=(r, l, L), r \geq R$ étant la distance $O G, l$ la longitude et $L$ la latitude, voir figure 3 i.

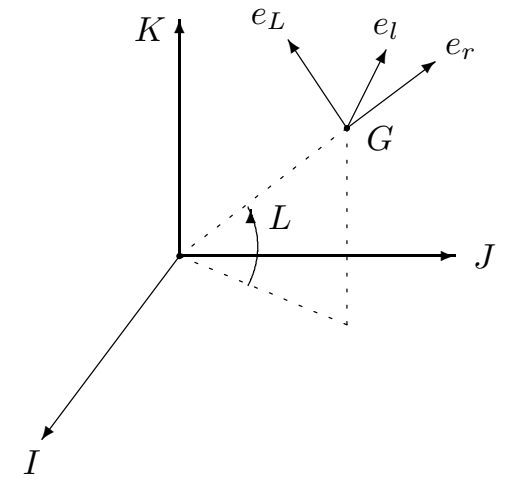

(i)

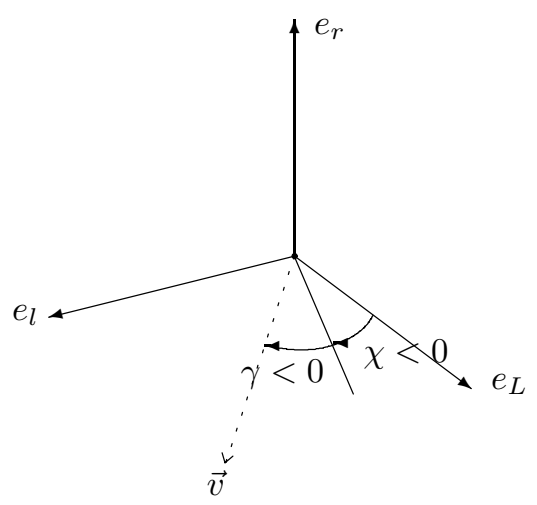

(ii)

FiguRE 3

Le système de coordonnées sphériques présente une singularité au pôle Nord et au pôle Sud. Pour écrire la dynamique sous forme plus simple on introduit le repère mobile $R_{2}=(i, j, k)$ dont l'origine est $G$ de la manière suivante. Soit $\zeta: t \mapsto(x(t), y(t), z(t))$ la trajectoire de $G$ mesurée dans le repère $R_{1}$ et $\vec{v}$ la vitesse relative $\vec{v}=\dot{x} I+\dot{y} J+\dot{z} K$. Pour définir $i$ on pose $: \vec{v}=|v| i$. Le vecteur $j$ est un vecteur unitaire du plan $\left(i, e_{r}\right)$ perpendiculaire à $i$ et orienté par $j . e_{r}>0$. On pose $k=i \wedge j$. La direction de la vitesse est paramétrisée dans le repère $R_{1}^{\prime}=\left(e_{r}, e_{l}, e_{L}\right)$ par deux angles, voir figure $3 \mathrm{ii}$ :

- la pente $\gamma$;

- l'azimut $\chi$.

L'équation fondamentale de la mécanique, qui est une équation différentielle du second ordre sur $\mathbb{R}^{3}$, se traduit par un système dans les coordonnées $(r, l, L, v, \gamma, \chi)$.

\subsection{Le modèle pour les forces}

Pour l'arc atmosphérique on fait les hypothèses suivantes :

Hypothèse 1 : la navette est un planeur, c'est-à-dire que la poussée de la navette est nulle.

Hypothèse 2 : on suppose que la vitesse de l'atmosphère est la vitesse de la Terre. La vitesse relative de la navette par rapport à la Terre est donc la vitesse relative $\vec{v}$.

Les forces agissant sur la navette sont de deux types :

- force de gravité : pour simplifier on suppose que la Terre est sphérique et que la force de gravité est 
orientée selon $e_{r}$. Dans le repère $R_{2}$ elle s'écrit :

$$
\vec{P}=-m g(i \sin \gamma+j \cos \gamma)
$$

où $g=g_{0} / r^{2}$.

- force aérodynamique : la force fluide due à l'atmosphère est une force $\vec{F}$ qui se décompose en :

- une composante dite de traînée opposée à la vitesse de la forme :

$$
\vec{D}=\left(\frac{1}{2} \rho S C_{D} v^{2}\right) i
$$

- une force dite de portance perpendiculaire à $\vec{v}$ donnée par :

$$
\vec{L}=\frac{1}{2} \rho S C_{L} v^{2}(j \cos \mu+k \sin \mu)
$$

où $\mu$ est l'angle de gîte, $\rho=\rho(r)$ est la densité de l'atmosphère, et $C_{D}, C_{L}$ sont respectivement les coefficients de traînée et de portance.

Hypothèse 3 : les coefficients $C_{D}$ et $C_{L}$ dépendent de l'angle d'attaque $\alpha$ qui est l'angle entre l'axe du planeur et le vecteur vitesse. C'est a priori un contrôle mais on suppose que durant l'arc atmosphérique il est fixé. Il est intéressant de noter que les simulations numériques de [3] où on autorise sa variation, il est constant le long de la trajectoire optimale.

Notre seul contrôle est donc l'angle de gîte $\mu$ dont l'effet est double : modifier l'altitude mais aussi tourner à droite ou à gauche.

\subsection{Le modèle pour l'atmosphère}

La densité atmosphérique est tabulée pour la Terre, Mars et Vénus et on choisit un modèle exponentiel :

$$
\rho(r)=\rho_{0} \mathrm{e}^{-\beta r}
$$

\subsection{Les équations du système}

L'arc atmosphérique est décrit par le système suivant :

$$
\begin{aligned}
\frac{\mathrm{d} r}{\mathrm{~d} t} & =v \sin \gamma \\
\frac{\mathrm{d} v}{\mathrm{~d} t} & =-g \sin \gamma-\frac{1}{2} \rho \frac{S C_{D}}{m} v^{2}+\Omega^{2} r \cos L(\sin \gamma \cos L-\cos \gamma \sin L \cos \chi) \\
\frac{\mathrm{d} \gamma}{\mathrm{d} t} & =\cos \gamma\left(-\frac{g}{v}+\frac{v}{r}\right)+\frac{1}{2} \rho \frac{S C_{L}}{m} v \cos \mu+2 \Omega \cos L \sin \chi+\Omega^{2} \frac{r}{v} \cos L(\cos \gamma \cos L+\sin \gamma \sin L \cos \chi) \\
\frac{\mathrm{d} L}{\mathrm{~d} t} & =\frac{v}{r} \cos \gamma \cos \chi \\
\frac{\mathrm{d} l}{\mathrm{~d} t} & =-\frac{v}{r} \frac{\cos \gamma \sin \chi}{\cos L} \\
\frac{\mathrm{d} \chi}{\mathrm{d} t} & =\frac{1}{2} \rho \frac{S C_{L}}{m} \frac{v}{\cos \gamma} \sin \mu+\frac{v}{r} \cos \gamma \tan L \sin \chi+2 \Omega(\sin L-\tan \gamma \cos L \cos \chi)+\Omega^{2} \frac{r}{v} \frac{\sin L \cos L \sin \chi}{\cos \gamma}
\end{aligned}
$$

où l'état est $q=(r, v, \gamma, l, L, \chi)$ et le contrôle est l'angle de gîte $\mu$. 
Coordonnées Kepleriennes. En supposant la Terre fixe $(\Omega=0)$ et que le système n'est soumis qu'à la force de gravitation, les trajectoires ont les propriétés suivantes :

Propriété 1 : Elles sont planes.

Propriété 2 : Si l'énergie est strictement négative, ce sont des ellipses dont le centre de la Terre est un foyer.

Ces propriétés sont la conséquence de l'existence d'intégrales premières qui sont :

( $\left.I_{1}\right)$ Moment cinétique $M=m x \wedge \dot{x}$, où $x$ est la position et $\dot{x}$ la vitesse, la conservation du moment cinétique impliquant que le mouvement est dans un plan normal à $M$.

$\left(I_{2}\right)$ Énergie totale $E=\frac{m \dot{x}^{2}}{2}-\frac{g_{0}}{r}$.

Il existe une autre intégrale première dite de Laplace due à la nature spécifique du potentiel :

(I $\left.I_{3}\right) L=\dot{x} \wedge M-g_{0} e_{r}$.

Le mouvement Keplerien est donc caractérisé par la normale au plan de l'ellipse $\frac{x \wedge \dot{x}}{|x \wedge \dot{x}|}$, l'angle $\omega$ du péricentre, la longueur du grand axe et l'excentricité de l'ellipse, voir figure 4i.

La position du satellite sur l'ellipse est caractérisée par un angle $\theta$ que l'on remplace en général par l'anomalie excentrique $\varphi$, voir figure 4ii.

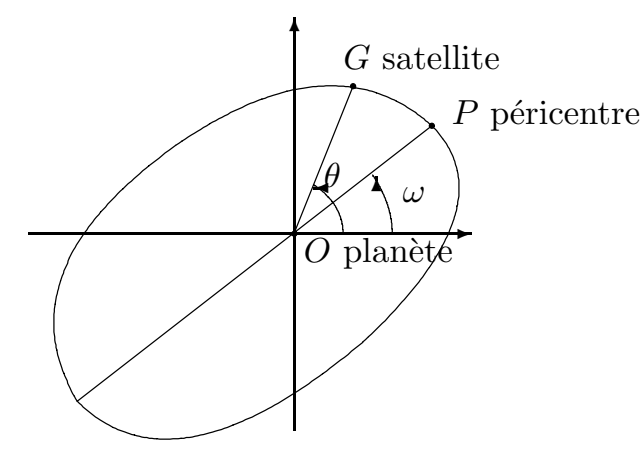

(i)

FiguRe 4

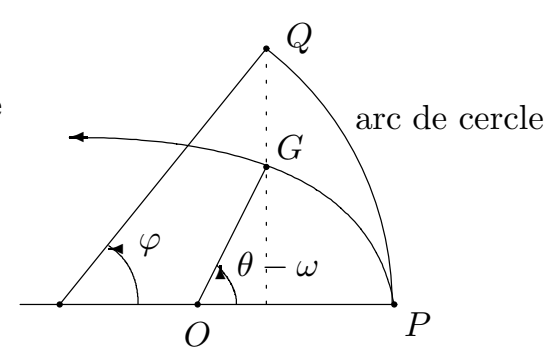

(ii)

On rappelle les relations suivantes :

$$
a=\frac{g_{0}}{2|E|} \quad e=\sqrt{1+2 \frac{E M^{2}}{m g_{0}^{2}}} .
$$

L'angle $\omega$ caractérisant le péricentre est plus complexe à calculer. Pour un mouvement général dans un champ central l'angle entre deux passages par un péricentre n'est pas en général constant et dans le problème de Kepler on doit pour le caractériser utiliser l'intégrale première de Laplace.

Notons $r_{0}=\left|\overrightarrow{O G}_{0}\right|$ la position initiale, $\vec{v}_{0}$ la vitesse initiale, $\alpha_{0}$ l'angle entre $\overrightarrow{O G}_{0}$ et $\vec{v}_{0}$, soit $C=r_{0} v_{0} \sin \alpha_{0}$, $\vec{j}_{0}$ vecteur unité perpendiculaire à $\overrightarrow{O P}_{0}$ dans le plan du mouvement, et $\vec{H}$ le vecteur défini par l'égalité :

$$
\vec{v}_{0}=-\frac{g_{0}}{C}\left(\vec{j}_{0}+\vec{H}\right)
$$

L'angle $\omega$ est défini par la propriété que l'angle entre $\vec{H}$ et $\overrightarrow{O P}$ est égal à $\pi / 2$.

L'évolution sur la trajectoire elliptique est donnée par l'équation de Kepler :

$$
\varphi-e \sin \varphi=\frac{2 \pi}{T}\left(t-t_{P}\right)
$$


où $t_{P}$ est l'instant de passage au péricentre, et $T=2 \pi a^{3 / 2} \sqrt{\frac{m}{g_{0}}}$ est la période de révolution. Définissons :

$$
\psi=(\varphi-e \sin \varphi) \frac{T}{2 \pi}
$$

Alors $\dot{\psi}=1$, et on obtient :

Proposition 2.1. Dans les coordonnées $q=\left(\frac{M}{\mid M}, a, e, \omega, \psi\right)$, l'équation de Kepler est linéaire $: K=\frac{\partial}{\partial \psi}$.

La dérive du système décrivant la navette est donc linéarisée dans un tel système de coordonnées. On appelle ellipse osculatrice à une trajectoire du système en un point $q_{1}$ l'ellipse du système libre passant par $q_{1}$. Le système de coordonnées Keplerien est bien adapté pour étudier l'action de la traînée qui est colinéaire à $\vec{v}$ et le plan contenant l'ellipse osculatrice est dans ce cas fixe et coïncide avec le plan osculateur contenant la vitesse et l'accélération. Par contre la force de portance est perpendiculaire à $\vec{v}$. Un choix de coordonnées canonique pour étudier le système est délicat.

\section{LE PROBlÈME DE CONTRÔLE OPTIMAL}

\subsection{Réduction du problème}

On va négliger dans notre problème le terme de Coriolis et d'entraînement, ce qui revient à négliger la rotation de la planète sur son axe. On peut alors adopter la simplification de [9] : le pôle nord n'a pas de signification intrinsèque et on peut supposer que le point final est sur l'axe NS de la Terre. Exploitant la symétrie des équations (4) où la longitude n'intervient pas dans la dynamique des coordonnées $(r, v, \gamma, L, \chi)$ et le fait que le point cible est une singularité du système de coordonnées, on se restreint au système :

$$
\begin{aligned}
\frac{\mathrm{d} r}{\mathrm{~d} t} & =v \sin \gamma \\
\frac{\mathrm{d} v}{\mathrm{~d} t} & =-g \sin \gamma-\frac{1}{2} \rho \frac{S C_{D}}{m} v^{2} \\
\frac{\mathrm{d} \gamma}{\mathrm{d} t} & =\cos \gamma\left(-\frac{g}{v}+\frac{v}{r}\right)+\frac{1}{2} \rho \frac{S C_{L}}{m} v \cos \mu \\
\frac{\mathrm{d} L}{\mathrm{~d} t} & =\frac{v}{r} \cos \gamma \cos \chi \\
\frac{\mathrm{d} \chi}{\mathrm{d} t} & =\frac{v}{r} \cos \gamma \tan L \sin \chi+\frac{1}{2} \rho \frac{S C_{L}}{m} \frac{v}{\cos \gamma} \sin \mu
\end{aligned}
$$

où l'état est $q=(r, v, \gamma, L, \chi)$.

Pour simplifier on fait les hypothèses suivantes :

Hypothèse $4: g=\frac{g_{0}}{r^{2}}$ est supposé constant. C'est une hypothèse quantitativement justifiée, par contre on perd les propriétés Kepleriennes du système.

Hypothèse 5 : Les coefficients de traînée et de finesse $C_{D}, C_{L}$ dépendent de l'angle d'attaque supposé fixé et du nombre de Machs, quotient de la vitesse de la navette et de la vitesse du son (qui dépend aussi de la densité de l'atmosphère). Ici l'hypothèse est très simplificatrice et doit être révisée ultérieurement.

\subsection{Contrôles et bornes sur le contrôle}

Le contrôle est soit $\mu$ soit $\dot{\mu}$. Dans le premier cas on peut imposer les bornes $\mu \in\left[-\frac{\pi}{2}, \frac{\pi}{2}\right]$ ou $\mu \in[-\pi, \pi]$. On pose $u_{1}=\cos \mu ; u_{1}$ contrôle la pente $\gamma$. On peut noter que le cahier des charges du CNES accepte des 
portances négatives, i.e. $u_{1} \in[-1,+1]$. On pose $u_{2}=\sin \mu ; u_{2}$ contrôle l'azimut et le signe de $u_{2}$ autorise le planeur à tourner à droite ou à gauche. Le système s'écrit donc soit sous la forme :

$$
\dot{q}=X(q)+u_{1} Y_{1}(q)+u_{2} Y_{2}(q), \quad u_{1}^{2}+u_{2}^{2}=1
$$

soit sous la forme :

$$
\dot{q}=X(q)+u Y(q), \quad|u| \leq M
$$

\subsection{Contraintes sur l'état}

C'est l'une des caractéristiques importantes du problème. Il faut prendre en compte trois contraintes sur l'état :

- Contrainte sur le flux thermique :

$$
\varphi=C_{q} \sqrt{\rho} v^{3} \leq \varphi^{\max }
$$

- Contrainte sur l'accélération normale :

$$
\gamma_{n}=\gamma_{n 0}(\alpha) \rho v^{2} \leq \gamma_{n}^{\max }
$$

- Contrainte sur la pression dynamique :

$$
\frac{1}{2} \rho v^{2} \leq P^{\max }
$$

Une seule des contraintes $(7,8)$ est à prendre en compte car $C_{D}, C_{L}$ sont constants.

\subsection{Le coût optimal}

Plusieurs choix sont possibles et les critères à prendre en compte sont le facteur d'usure lié à l'intégrale du flux thermique et le confort de vol lié à l'intégrale de l'accélération normale. On prend le premier critère, et le problème est de minimiser :

$$
J(\mu)=\int_{0}^{T} C_{q} \sqrt{\rho} v^{3} \mathrm{~d} t
$$

le temps $T$ de l'arc atmosphérique étant libre. On introduit l'équation :

$$
\frac{\mathrm{d} \tilde{q}_{0}}{\mathrm{~d} t}=C_{q} \sqrt{\rho} v^{3}
$$

avec $\tilde{q}_{0}(0)=0$.

\subsection{Conditions frontières}

Le temps de transfert $T$ est libre et l'on a deux choix pour les conditions limites :

- conditions limites fixées à $t=0$ et $t=T$ pour $q=(r, v, \gamma, l, \chi)$ (la latitude est prise en compte en normalisant la cible au pôle nord) ;

- $\gamma \in\left[\gamma_{\min }, \gamma_{\max }\right]$ à $t=0$, avec l'hypothèse qu'un ajustement préliminaire est possible en utilisant la poussée sur l'arc Keplerien. 


\subsection{Quelques données numériques sur l'arc atmosphérique}

Pour fixer les idées dans le problème de rentrée sur Terre, les valeurs numériques caractéristiques du domaine de vol sont les suivantes :

- Altitude $h=r-R \in[40 \mathrm{~km}, 120 \mathrm{~km}]$.

- Module de la vitesse $v \in[2000 \mathrm{~m} / \mathrm{s}, 8000 \mathrm{~m} / \mathrm{s}]$ (rentrée à grande vitesse).

- Pente de la trajectoire $-15^{\circ}<\gamma<0$.

\subsection{La notion de trajectoire équilibrée}

C'est un concept important dans la littérature spatiale que l'on peut traduire ainsi. Considérons l'équation d'évolution de la pente :

$$
\frac{\mathrm{d} \gamma}{\mathrm{d} t}=\cos \gamma\left(-\frac{g}{v}+\frac{v}{r}\right)+\frac{1}{2} \rho \frac{S C_{L}}{m} v \cos \mu
$$

Le domaine de vol équilibré est l'ensemble des conditions initiales tel que $0 \in\left[\dot{\gamma}_{u_{1}=-1}, \dot{\gamma}_{u_{1}=+1}\right]$ avec $u_{1}=\cos \mu$. Avec $\cos \gamma \sim 1$ et en négligeant le terme en $\frac{v}{r}$ on obtient la condition :

$$
\frac{1}{2} \rho \frac{S C_{L}}{m}>\frac{g}{v^{2}}
$$

(voir Fig. 5).

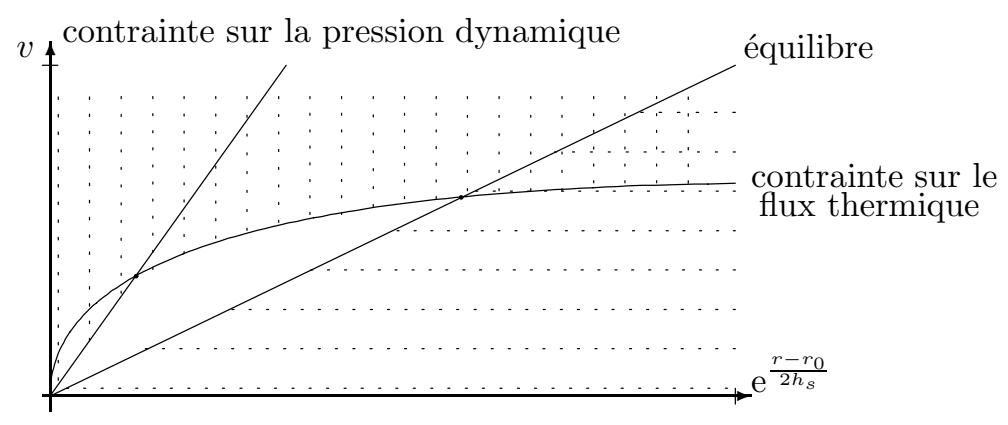

FiguRE 5

Cette condition n'est pas toujours réalisée, en particulier en début de trajectoire, car il faut que la vitesse soit assez petite pour que la trajectoire soit elliptique : $E=\frac{1}{2} m v^{2}-\frac{g_{0}}{r}<0$. Par ailleurs le domaine de vol équilibré dépend de la densité de l'atmosphère (faible en début de trajectoire) et inversement proportionnelle à la masse. C'est une condition de contrôlabilité cruciale qui signifie que la portance peut équilibrer le terme de gravité.

On peut observer que pour $\mu=k \pi$ la portance est contenue dans le plan de l'ellipse osculatrice et le mouvement est plan. On doit le simuler pour $u_{1}=+1$ (portance maximale) et $u_{1}=-1$ (portance minimale).

\section{ACCessibilité et COURBes extrémales POUR LE Système nON CONTRAint}

\section{1. Énoncé du problème et notations}

On considère un système mono-entrée :

$$
\dot{q}=f(q, u), q \in D, u \in \mathcal{U}
$$


et un coût à minimiser de la forme :

$$
J(u)=\int_{0}^{T} \varphi(q) \mathrm{d} t
$$

où le temps de transfert $T$ est libre et la fonction $\varphi$ ne dépend pas de $u$. L'ensemble des contrôles admissibles est l'ensemble $\mathcal{U}$ des applications mesurables $u:[0, T] \rightarrow U$. L'espace d'états $D$ est un domaine de $\mathbb{R}^{n}$ avec des contraintes :

- Contrainte sur le flux thermique :

$$
c_{1}(q)=\varphi(q) \leq \alpha_{1} .
$$

- Contrainte sur l'accélération normale :

$$
c_{2}(q)=\gamma_{n}(q) \leq \alpha_{2}
$$

- Contrainte sur le facteur de charge :

$$
c_{3}(q) \leq \alpha_{3}
$$

où $q=(r, v, \gamma, \chi, L)$ et les contraintes ne portent que sur $(r, v)$.

Les conditions limites sont de la forme:

$$
q(0)=q_{0} \text { et } q(T)=q_{1} \text { fixés }
$$

ou bien

$$
q=(r, v, \gamma, \chi, L) \text { avec } \gamma(0) \in\left[\gamma_{1}, \gamma_{2}\right], \gamma_{1}<\gamma_{2} \leq 0 .
$$

On note $q\left(t, u, q_{0}\right)$ la trajectoire du système associée à $u \in \mathcal{U}$ et issue en $t=0$ de $q_{0}, R\left(q_{0}, t\right)=\bigcup_{u \in \mathcal{U}} q\left(t, u, q_{0}\right)$ l'ensemble des états accessibles au temps $t$ fixé, et $R\left(q_{0}\right)=\bigcup_{t \text { assez petit }} R\left(q_{0}, t\right)$ l'ensemble des états accessibles en temps petit.

\subsection{Le Principe du Minimum}

On rappelle le Principe du Minimum [18] qui permet de paramétriser la frontière de l'ensemble des états accessibles pour le système augmenté :

$$
\dot{q}=f(q, u), \dot{\tilde{q}}_{0}=\varphi(q)
$$

On introduit le Hamiltonien :

$$
H(q, p, u)=\langle p, f(q, u)\rangle+\tilde{p}_{0} \varphi(q)
$$

où $q=(r, v, \gamma, L, \chi), p=\left(p_{r}, p_{v}, p_{\gamma}, p_{L}, p_{\chi}\right)$ est le vecteur adjoint, et $\tilde{p}_{0}$ est une constante telle que $\left(p, \tilde{p}_{0}\right) \neq(0,0)$.

Définition 4.1. Si $\tilde{p}_{0} \neq 0$, on est dans le cas normal, et si $\tilde{p}_{0}=0$ on est dans le cas anormal.

Définition 4.2. On appelle extrémale un triplet $(q, p, u)$ solution du Principe du Minimum :

$$
\begin{gathered}
\dot{q}=f(q, u)=\frac{\partial H}{\partial p} \\
\dot{p}=-p \frac{\partial f}{\partial q}(q, u)-\tilde{p}_{0} \frac{\partial \varphi}{\partial q}=-\frac{\partial H}{\partial q} \\
H(q, p, u)=\min _{w \in \mathcal{U}} H(q, p, w) .
\end{gathered}
$$


Proposition 4.3. Une solution optimale du problème non contraint est une projection sur l'espace d'états d'une solution extrémale. De plus puisque le temps de transfert $T$ est libre, une extrémale est exceptionnelle, c'està-dire contenue dans le niveau d'énergie $H=0$. De plus $\tilde{p}_{0} \geq 0$. Si $\gamma$ est libre à l'instant initial, le vecteur adjoint $p$ satisfait la condition de transversalité :

$$
\left.p_{\gamma}(0)=0 \text { si } \gamma(0) \in\right] \gamma_{1}, \gamma_{2}[\text {. }
$$

Le lien avec la frontière de l'ensemble d'états accessibles est le suivant :

Proposition 4.4. Soit $\tilde{q}(t)=\left(q(t), \tilde{q}_{0}(t)\right)$ une trajectoire du système augmenté issue en $t=0$ de $\tilde{q}(0)=\left(q_{0}, 0\right)$ et $\tilde{R}$ l'ensemble des états accessibles du système augmenté. Soit $\tilde{q}_{1}$ un point frontière de $\tilde{R}, \tilde{q}_{1}=\tilde{q}\left(t_{1}\right)$. Alors la trajectoire $t \mapsto \tilde{q}(t)$ est une extrémale et de plus elle est contenue dans $H=0$.

\subsection{Définition du sous-système I}

En négligeant la vitesse de rotation de la planète, le système décrivant l'évolution de la navette peut se décomposer en :

$$
\dot{q}_{1}=f_{1}\left(q_{1}, u_{1}\right), \dot{q}_{2}=f_{2}\left(q, u_{2}\right)
$$

où $q_{1}=(r, v, \gamma), q_{2}=(\chi, L), u_{1}=\cos \mu, u_{2}=\sin \mu$.

Le système adjoint (18) se décompose alors en :

$$
\left(\begin{array}{ll}
\dot{p}_{1} \dot{p}_{2} 0
\end{array}\right)=-\left(\begin{array}{llll}
p_{1} & p_{2} & \tilde{p}_{0}
\end{array}\right)\left(\begin{array}{ccc}
\frac{\partial f_{1}}{\partial q_{1}} & 0 & 0 \\
\frac{\partial f_{2}}{\partial q_{1}} & \frac{\partial f_{2}}{\partial q_{2}} & 0 \\
\frac{\partial \varphi}{\partial q_{1}} & 0 & 0
\end{array}\right) .
$$

Si l'on oublie la condition terminale sur $q_{2}=(\chi, L)$, on déduit de la relation de transversalité $: p_{2}(T)=0$, et donc $p_{2}(t)=0$ pour tout $t$. L'analyse des extrémales se réduit donc à l'analyse des solutions de :

où $H$ est le Hamiltonien réduit :

$$
\begin{gathered}
\dot{q}_{1}=\frac{\partial H}{\partial p_{1}}, \dot{p}_{1}=-\frac{\partial H}{\partial q_{1}} \\
H\left(p_{1}, q_{1}, u_{1}\right)=\min _{|w| \leq 1} H\left(p_{1}, q_{1}, w\right)
\end{gathered}
$$

$$
H\left(p_{1}, q_{1}, u_{1}\right)=\left\langle p_{1}, f_{1}\left(q, u_{1}\right)\right\rangle, u_{1}=\cos \mu .
$$

C'est le système extrémal associé au problème $\min \int_{0}^{T} \varphi(\rho, v) \mathrm{d} t$ pour le sous-système I défini par :

$$
\begin{aligned}
& \frac{\mathrm{d} r}{\mathrm{~d} t}=v \sin \gamma \\
& \frac{\mathrm{d} v}{\mathrm{~d} t}=-g \sin \gamma-\frac{1}{2} \rho \frac{S C_{D}}{m} v^{2} \\
& \frac{\mathrm{d} \gamma}{\mathrm{d} t}=\cos \gamma\left(-\frac{g}{v}+\frac{v}{r}\right)+\frac{1}{2} \rho \frac{S C_{L}}{m} v \cos \mu .
\end{aligned}
$$

On peut observer que seule la variable $q_{1}=(r, v, \gamma)$ apparaît dans les contraintes d'état de la navette. On va concentrer la première étape de l'analyse du problème optimal sur le sous-système I. 


\subsection{Analyse des extrémales du sous-système I}

\subsubsection{Réduction du problème et définitions}

Considérons le système affine et mono-entrée :

$$
\dot{q}=X+u Y,|u| \leq 1
$$

et un coût à minimiser de la forme :

$$
J(u)=\int_{0}^{T} \varphi(q) \mathrm{d} t
$$

Supposons $\varphi(q)>0$ dans le domaine d'état. On introduit l'équation :

$$
\dot{\tilde{q}}_{0}=\varphi(q), \quad \tilde{q}_{0}(0)=0
$$

et $\tilde{q}=\left(q, \tilde{q}_{0}\right)$ l'espace d'états élargi. Les équations (22) et (23) définissent donc le système élargi :

$$
\dot{\tilde{q}}=\tilde{X}(\tilde{q})+u \tilde{Y}(\tilde{q})
$$

On introduit un nouveau paramétrage $s$ des équations en posant :

$$
\mathrm{d} s=\varphi(q(t)) \mathrm{d} t
$$

et en notant ' la dérivée par rapport à $s$, le système (22) s'écrit :

$$
q^{\prime}=\bar{X}(q)+u \bar{Y}(q),|u| \leq 1
$$

où $\bar{X}=\psi X, \bar{Y}=\psi Y$, et $\psi=\frac{1}{\varphi}$. Le problème de contrôle équivaut alors à un problème de temps minimal.

On va analyser l'existence de trajectoires singulières pour le problème.

Définition 4.5. Considérons le système $\dot{q}=X+u Y$ noté $(X, Y)$. Une trajectoire singulière est la projection sur l'espace d'états des équations :

$$
\dot{q}=\frac{\partial H}{\partial p}, \dot{p}=-\frac{\partial H}{\partial q},\langle p, Y\rangle=0
$$

où $H=\langle p, X+u Y\rangle$. Elle est dite exceptionnelle si $H=0$, admissible si $|u| \leq 1$ et strictement admissible si $|u|<1$.

Notation. Si $X_{1}, X_{2}$ sont deux champs de vecteurs, on note $\left[X_{1}, X_{2}\right]$ le crochet de Lie calculé avec la convention :

$$
\left[X_{1}, X_{2}\right](q)=\frac{\partial X_{2}}{\partial q}(q) X_{1}(q)-\frac{\partial X_{1}}{\partial q}(q) X_{2}(q) .
$$

Proposition 4.6. Dans le domaine de vol où $\cos \gamma \neq 0$, il n'y a pas d'arc singulier exceptionnel pour le système $(X, Y)$.

Démonstration. Les extrémales singulières sont contenues dans $\langle p, Y(q)\rangle=0$. En dérivant deux fois par rapport à $t$ il vient :

$$
\begin{aligned}
& \langle p,[X, Y](q)\rangle=0 \\
& \langle p,[X,[X, Y]](q)\rangle+u\langle p,[Y,[X, Y]](q)\rangle=0 .
\end{aligned}
$$


Calculons les crochets de Lie des relations avec :

$$
X=v \sin \gamma \frac{\partial}{\partial r}-\left(g \sin \gamma+k \rho v^{2}\right) \frac{\partial}{\partial v}+\cos \gamma\left(-\frac{g}{v}+\frac{v}{r}\right) \frac{\partial}{\partial \gamma}, \quad Y=k^{\prime} \rho v \frac{\partial}{\partial \gamma}
$$

où $k=\frac{1}{2} \frac{S C_{D}}{m}, k^{\prime}=\frac{1}{2} \frac{S C_{L}}{m}$ et on a supposé que $g=\frac{g_{0}}{r^{2}} \sim$ cste et que $k, k^{\prime}$ sont des constantes. Le concept de trajectoire singulière est invariant par feedback et dans nos calculs on peut donc remplacer $X, Y$ par :

$$
X=v \sin \gamma \frac{\partial}{\partial r}-\left(g \sin \gamma+k \rho v^{2}\right) \frac{\partial}{\partial v}, \quad Y=\frac{\partial}{\partial \gamma}
$$

On obtient donc :

$$
\begin{aligned}
{[X, Y] } & =-v \cos \gamma \frac{\partial}{\partial r}+g \cos \gamma \frac{\partial}{\partial v} \\
{[Y,[X, Y]] } & =v \sin \gamma \frac{\partial}{\partial r}-g \sin \gamma \frac{\partial}{\partial v}
\end{aligned}
$$

donc $[X, Y]$ et $[Y,[X, Y]]$ sont colinéaires. De plus :

$$
[X,[X, Y]]=k \rho v^{2} \cos \gamma \frac{\partial}{\partial r}+\left(-k \rho v^{3} \cos \gamma+2 k \rho g v \cos \gamma\right) \frac{\partial}{\partial v}
$$

Les extrémales singulières sont situées sur $\Sigma^{\prime}:\langle p, Y\rangle=\langle p,[X, Y]\rangle=0$, c'est-à-dire $: p_{\gamma}=p_{v} g-p_{r} v=0$. On introduit :

$$
\begin{aligned}
D & =\operatorname{det}(Y,[X, Y],[Y,[X, Y]]) \\
D^{\prime} & =\operatorname{det}(Y,[X, Y],[X,[X, Y]]) \\
D^{\prime \prime} & =\operatorname{det}(Y,[X, Y], X) .
\end{aligned}
$$

Il résulte de nos calculs que les arcs singuliers sont situés sur $D=D^{\prime}=0$, et de plus s'ils sont exceptionnels on doit avoir $D^{\prime \prime}=0$. Or :

$$
\begin{aligned}
D & =0 \\
D^{\prime} & =k v^{2} \cos ^{2} \gamma\left(\rho^{\prime} v^{2}-3 \rho g\right) \\
D^{\prime \prime} & =k \rho v^{3} \cos \gamma
\end{aligned}
$$

Puisque $\cos \gamma \neq 0$, la proposition est prouvée.

Les calculs montrent par ailleurs que le système (21), sous-système I de la navette, a les propriétés suivantes :

Lemme 4.7. Si $\cos \gamma \neq 0$ alors :

1. $X, Y,[X, Y]$ sont indépendants et forment un repère ;

2. $[Y,[X, Y]] \in \operatorname{Vect}\{Y,[X, Y]\}$.

On a donc montré que le sous-système I n'admet pas d'extrémales anormales, c'est-à-dire indépendantes du coût $\left(\tilde{p}_{0}=0\right)$. On va étudier l'existence d'extrémales singulières et classifier localement les extrémales.

\subsubsection{Analyse des extrémales}

Considérons le problème du temps minimal pour le système (26) :

$$
q^{\prime}=\bar{X}(q)+u \bar{Y}(q),|u| \leq 1 .
$$

Introduisons quelques définitions. 
Définition 4.8. L'ensemble $\Sigma:\langle p, \bar{Y}\rangle=0$ s'appelle la surface de commutation. Soit $(q, p, u)$ une extrémale définie sur $[0, T]$. Elle est dite singulière si elle est contenue dans $\Sigma$, bang si $u=+1$ ou -1 , et bang-bang si $u(t)$ est constant par morceaux et défini presque partout par $u(t)=-$ signe $\langle p(t), \bar{Y}(q(t))\rangle$. On note respectivement $\gamma_{+}, \gamma_{-}, \gamma_{s}$ un arc lisse associé à $u=+1, u=-1$ ou $u$ contrôle singulier, et $\gamma_{1} \gamma_{2}$ représente un arc $\gamma_{1}$ suivi d'un $\operatorname{arc} \gamma_{2}$.

Calculons les crochets de Lie :

$$
\bar{X}=\psi\left(v \sin \gamma \frac{\partial}{\partial r}-\left(g \sin \gamma+k \rho v^{2}\right) \frac{\partial}{\partial v}+\cos \gamma\left(-\frac{g}{v}+\frac{v}{r}\right) \frac{\partial}{\partial \gamma}\right), \quad \bar{Y}=\psi k^{\prime} \rho v \frac{\partial}{\partial \gamma}
$$

avec $\psi=\frac{1}{\varphi}$. En utilisant la formule suivante où $f_{1}, f_{2}$ sont des fonctions lisses :

$$
\left[f_{1} X, f_{2} Y\right]=f_{1} f_{2}[X, Y]+f_{1}\left(X . f_{2}\right) Y-f_{2}\left(Y . f_{1}\right) X
$$

où $Z . f=\frac{\partial f}{\partial q} Z(q)$ est la dérivée de Lie, il vient :

$$
[\bar{X}, \bar{Y}]=\psi^{2}[X, Y]+\psi(X . \psi) Y-\psi(Y . \psi) X
$$

Puisque $Y=k^{\prime} \rho v \frac{\partial}{\partial \gamma}$ et $\psi$ ne dépend que de $\rho, v$, on a $Y . \psi=0$. Donc :

$$
[\bar{X}, \bar{Y}]=\psi^{2}[X, Y]+\psi(X . \psi) Y
$$

En calculant $[\bar{Y},[\bar{X}, \bar{Y}]]$ de la même façon on obtient :

\section{Lemme 4.9.}

1. L'ensemble $\Sigma^{\prime}:\langle p, \bar{Y}\rangle=\langle p,[\bar{X}, \bar{Y}]\rangle=0$ coïncide avec $:\langle p, Y\rangle=\langle p,[X, Y]\rangle=0$.

2. $[\bar{Y},[\bar{X}, \bar{Y}]]=\psi^{3}[Y,[X, Y]] \bmod \operatorname{Vect}\{Y,[X, Y]\}$, et $\operatorname{donc}[\bar{Y},[\bar{X}, \bar{Y}]] \in \operatorname{Vect}\{Y,[X, Y]\}$.

De plus :

$$
\begin{aligned}
\bar{D} & =\operatorname{det}(\bar{Y},[\bar{X}, \bar{Y}],[\bar{Y},[\bar{X}, \bar{Y}]])=0 \\
\bar{D}^{\prime \prime} & =\operatorname{det}(\bar{Y},[\bar{X}, \bar{Y}], \bar{X})=\frac{k k^{\prime 2} \rho \cos \gamma}{C_{q}^{4} v^{7}}
\end{aligned}
$$

et donc $\bar{Y},[\bar{X}, \bar{Y}], \bar{X}$ forment un repère dans le domaine de vol où $\cos \gamma \neq 0$. Donc il existe des fonctions $a, b, c$ telles que :

$$
[\bar{X},[\bar{X}, \bar{Y}]]=a \bar{X}+b \bar{Y}+c[\bar{X}, \bar{Y}]
$$

Des calculs longs conduisent au résultat crucial suivant :

Lemme 4.10. Si $\cos \gamma \neq 0$, on $a$ :

1. $\bar{D}^{\prime}=\operatorname{det}(\bar{Y},[\bar{X}, \bar{Y}],[\bar{X},[\bar{X}, \bar{Y}]])=-\frac{\beta}{2} \frac{k k^{\prime 3} \rho \cos ^{2} \gamma}{C_{q}^{6} v^{11}} \neq 0$;

2. $a=-\frac{\beta}{2} \frac{k^{\prime} \sqrt{\rho}}{C_{q}^{2} v^{4}} \cos \gamma<0$.

Corollaire 4.11. Si $\cos \gamma \neq 0$, il n'existe pas de trajectoires singulières. 


\subsubsection{Application à la classification des extrémales au voisinage de $\Sigma$}

Soit $(q, p, u)$ une extrémale lisse définie sur $[0, T]$. En dérivant par rapport à $t$ la fonction de commutation $\phi: t \mapsto\langle p(t), \bar{Y}(q(t))\rangle$ on obtient :

$$
\begin{aligned}
\dot{\phi}(t) & =\langle p(t),[\bar{X}, \bar{Y}](q(t))\rangle \\
\ddot{\phi}(t) & =\langle p(t),[\bar{X},[\bar{X}, \bar{Y}]](q(t))+u(t)[\bar{Y},[\bar{X}, \bar{Y}]](q(t))\rangle .
\end{aligned}
$$

On utilise les résultats de [14] pour classifier les extrémales au voisinage d'un point $z_{0}=\left(q_{0}, p_{0}\right)$ de la surface de commutation.

1. Points ordinaires : $z_{0}$ appartient à $\Sigma:\langle p, \bar{Y}\rangle=0$. Supposons $\Sigma$ lisse et de codimension 1 en $z_{0}$. Si $\langle p,[\bar{X}, \bar{Y}]\rangle \neq 0$ en $z_{0}$, on dit que $z_{0}$ est un point de commutation ordinaire et chaque courbe extrémale dans un voisinage de $z_{0}$ est de la forme $\gamma_{+} \gamma_{-}$ou $\gamma_{-} \gamma_{+}$.

2. Points plis : $z_{0}$ appartient à $\Sigma^{\prime}:\langle p, \bar{Y}\rangle=\langle p,[\bar{X}, \bar{Y}]\rangle=0$. Si $\Sigma, \Sigma^{\prime}$ sont lisses et de codimensions respectives 1 et 2 , on dit que $z_{0}$ est un point pli. Si $(q, p, u)$ est une extrémale lisse passant par $z_{0}$, la fonction de commutation vérifie en $z_{0}$ :

et de plus :

$$
\phi(t)=\dot{\phi}(t)=0
$$

$$
\ddot{\phi}(t)=\langle p(t),[\bar{X},[\bar{X}, \bar{Y}]](q(t))+u(t)[\bar{Y},[\bar{X}, \bar{Y}]](q(t))\rangle
$$

et se réduit à $\langle p(t),[\bar{X},[\bar{X}, \bar{Y}]](q(t))\rangle$ d'après le lemme 4.9. De plus $\ddot{\phi}$ est non nulle sur $\Sigma$ d'après le lemme 4.10 et son signe est celui de $a\langle p, \bar{X}\rangle$. Donc chaque courbe correspondant à $u=+1$ ou $u=-1$ a un contact d'ordre 2 avec $\Sigma$ et chaque courbe extrémale au voisinage de $z_{0}$ est représentée sur la figure 6 . En utilisant la classification de [14] le point $z_{0}$ est un point parabolique, et chaque extrémale est localement bang-bang et de la forme $\gamma_{+} \gamma_{-} \gamma_{+}$ ou $\gamma_{-} \gamma_{+} \gamma_{-}$.

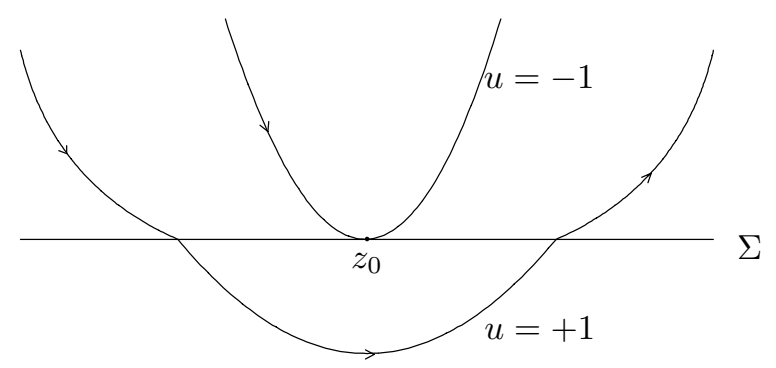

Figure 6. Solutions extrémales $(a<0)$.

En utilisant cette analyse et le Principe du Minimum on peut résoudre localement le problème de synthèse optimale.

Théorème 4.1. Si $\cos \gamma \neq 0$, chaque trajectoire temps minimale est localement de la forme $\gamma_{-} \gamma_{+} \gamma_{-}$, où $\gamma_{+}$

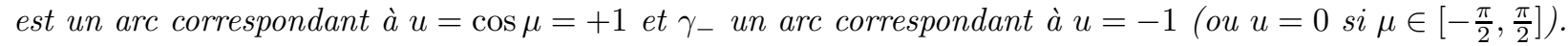

Démonstration. D'après le Principe du Minimum un arc optimal est extrémal et doit satisfaire :

$$
H=\langle p, \bar{X}(q)+u \bar{Y}(q)\rangle+p_{0}=0, p_{0} \geq 0 .
$$

Donc en $z_{0} \in \Sigma^{\prime}$, le vecteur adjoint est orienté avec la convention : $\langle p, \bar{X}(q)\rangle \leq 0$. Or :

$$
[\bar{X},[\bar{X}, \bar{Y}]]=a \bar{X}+b \bar{Y}+c[\bar{X}, \bar{Y}], a<0 .
$$


Donc seule une trajectoire extrémale de la forme $\gamma_{-} \gamma_{+} \gamma_{-}$peut être optimale. L'assertion est prouvée.

En changeant le problème du temps minimal en temps maximal on en déduit :

Corollaire 4.12. Si $\cos \gamma \neq 0$, chaque trajectoire temps maximale est localement de la forme $\gamma_{+} \gamma_{-} \gamma_{+}$.

Par ailleurs :

Corollaire 4.13. Chaque trajectoire optimale pour le sous-système I est bang-bang. De plus, il existe une borne uniforme $N$ sur le nombre de commutations des trajectoires optimales dans le domaine de vol.

\subsubsection{Géométrie de l'ensemble des états accessibles en temps petit}

On peut traduire les résultats précédents sur l'ensemble des états accessibles. En effet considérons le système en dimension 3 :

$$
\frac{\mathrm{d} q}{\mathrm{~d} s}=\bar{X}(q)+u \bar{Y}(q)
$$

et son extension en dimension 4 en adjoignant le coût, ici le temps. Soit $q_{0} \in \mathbb{R}^{3}$; on note $R\left(q_{0}\right)$ l'ensemble des états accessibles en temps petit, et $\tilde{R}\left(q_{0}, 0\right)$ l'ensemble des états accessibles en temps petit pour le système augmenté. Notre étude permet de le décrire, et s'inscrit dans le programme de [13,19, 20] pour classifier ces ensembles en s'appuyant sur des idées originales de Lobry, ceci en petite dimension. Le résultat suivant est basique.

Lemme 4.14. Considérons le système $(\bar{X}, \bar{Y})$ en dimension 3, et posons $g_{1}=\bar{X}+\bar{Y}, g_{2}=\bar{X}-\bar{Y}$. Supposons que $g_{1}, g_{2}$ et $\left[g_{1}, g_{2}\right]$ sont indépendants en $q_{0}$. Alors $R\left(q_{0}\right)$ est borné par les deux surfaces $S_{1}: \bigcup \gamma_{+} \gamma_{-}\left(q_{0}\right)$ et $S_{2}: \bigcup \gamma_{-} \gamma_{+}\left(q_{0}\right)$, et de plus $R\left(q_{0}\right)=\bigcup \gamma_{+} \gamma_{-} \gamma_{+}\left(q_{0}\right)\left(\right.$ ou $\left.\bigcup \gamma_{-} \gamma_{+} \gamma_{-}\left(q_{0}\right)\right)$.

En particulier un point $q_{1}$ à l'intérieur de $R\left(q_{0}\right)$ est accessible par un arc $\gamma_{+} \gamma_{-} \gamma_{+}\left(q_{0}\right)$ et un arc $\gamma_{-} \gamma_{+} \gamma_{-}\left(q_{0}\right)$ avec deux commutations. Notre étude montre que dans notre configuration de crochets de Lie, une des politiques est temps minimale et l'autre temps maximale. Cela se traduit en une description de la frontière de l'ensemble des états accessibles pour le système augmenté, et en particulier on a le résultat suivant, à comparer avec [19] :

Lemme 4.15. Si $\cos \gamma \neq 0$, la frontière de l'ensemble des états accessibles en temps petit du système augmenté $\tilde{R}\left(q_{0}, 0\right)$ est l'union de trajectoires $\tilde{\gamma}_{-} \tilde{\gamma}_{+} \tilde{\gamma}_{-}\left(q_{0}, 0\right)$ et $\tilde{\gamma}_{+} \tilde{\gamma}_{-} \tilde{\gamma}_{+}\left(q_{0}, 0\right)$ où $\tilde{\gamma}$ désigne une trajectoire du système augmenté.

Remarque 4.16. On peut rapprocher ce résultat de l'exemple en dimension 1 de l'introduction représenté sur la figure 1, où la frontière décrit les politiques temps minimal et temps maximal. Un des problèmes de notre étude est d'étendre cette description à la dimension 5 dans la configuration locale de l'algèbre de Lie de la navette, ce qui nous amène à la discussion de la section suivante.

\subsection{Accessibilité et contrôle optimal pour l'arc atmosphérique non contraint}

Le système s'écrit :

$$
\frac{\mathrm{d} q}{\mathrm{~d} s}=\bar{X}(q)+u_{1} \bar{Y}_{1}(q)+u_{2} \bar{Y}_{2}(q)
$$

où $q=(r, v, \gamma, \chi, L)$ en paramétrisant les trajectoires par le flux thermique. On a $u_{1}^{2}+u_{2}^{2}=1$, et on note $q^{\prime}=\bar{f}(q, \mu)$ le système mono-entrée où le contrôle est l'angle de gîte. On peut l'étendre de façon affine dans $\mathbb{R}^{6}$ en adjoignant l'équation $\dot{\mu}=v$.

Définition 4.17. On appelle problème optimal convexifié le problème où la contrainte $u_{1}^{2}+u_{2}^{2}=1$ est remplacée par la contrainte convexe $u_{1}^{2}+u_{2}^{2} \leq 1$.

L'analyse du problème convexifié est cruciale car les théorèmes d'existence de trajectoires optimales s'appliquent dans ce cadre. La procédure est donc d'analyser les extrémales du problème convexifié pour en déduire les trajectoires optimales de ce problème, puis de vérifier si elles satisfont la contrainte initiale $u_{1}^{2}+u_{2}^{2}=1$. Par ailleurs le problème convexifié où les deux contrôles $u_{1}, u_{2}$ agissent de façon indépendante a des propriétés géométriques intéressantes que l'on explique. 


\subsubsection{Géométrie du problème convexifié}

Contrôle $\mathbf{u}_{2}=\mathbf{0}$. On a alors un système mono-entrée : $\dot{q}=X+u_{1} Y_{1}$, dont la projection sur l'espace $(r, v, \gamma)$ a été étudiée en section 4.4. Dans ce cas, la force de portance est tangente au plan de la trajectoire du système libre, et l'algèbre de Lie engendrée par $X, Y_{1}$ est de dimension $\leq 4$. La réduction suivante est cruciale :

$$
\begin{aligned}
\frac{\mathrm{d} \chi}{\mathrm{d} t} & =\frac{v}{r} \cos \gamma \sin \chi \tan L \\
\frac{\mathrm{d} L}{\mathrm{~d} t} & =\frac{v}{r} \cos \gamma \cos \chi
\end{aligned}
$$

donc :

et la relation :

$$
\frac{\mathrm{d} \chi}{\mathrm{d} L}=\tan \chi \tan L
$$

$$
\int_{\chi(0)}^{\chi} \frac{\mathrm{d} \chi}{\tan \chi}=\int_{L(0)}^{L} \tan L \mathrm{~d} L .
$$

En particulier l'évolution $L \mapsto \chi(L)$ ne dépend pas du contrôle. En transférant le système de $L(0)$ à la latitude finale normalisée à $\frac{\pi}{2}$ (la cible est identifiée au pôle nord), on transfère donc $\chi$ de $\chi(L(0))$ à $\chi\left(\frac{\pi}{2}\right)$. Notons $\Pi$ la projection dans le plan $(r, v, \gamma)$. La projection par $\mathrm{d} \Pi$ du système est le sous-système I étudié en section 4.4 . On a donc la représentation de la figure 7.

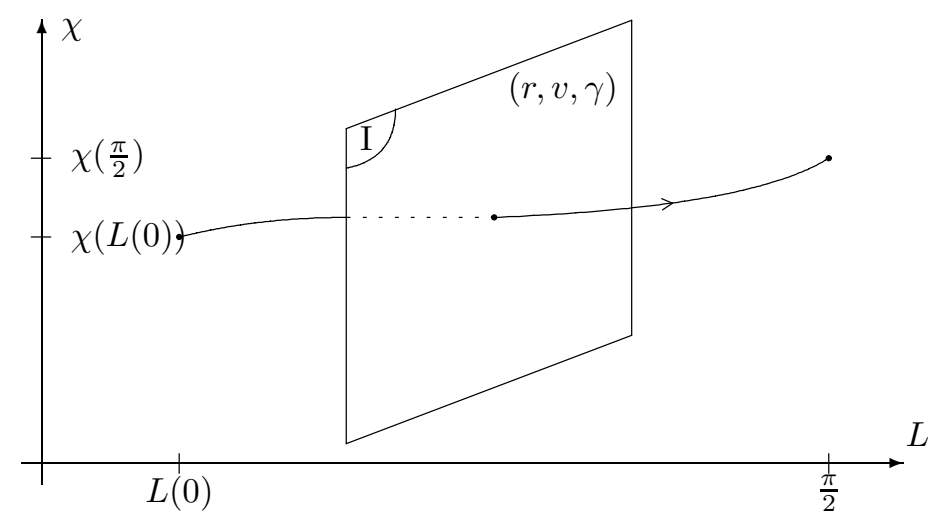

FIGURE 7

Or on a :

$$
\frac{\mathrm{d} L}{\cos \chi}=\frac{v}{r} \cos \gamma \mathrm{d} t
$$

$L$ étant croissante si $\chi \in]-\frac{\pi}{2}, \frac{\pi}{2}\left[\right.$. En paramétrant les trajectoires de I par $\mathrm{d} s=\frac{\mathrm{d} L}{\cos \chi}$, le système I devient :

$$
\frac{\mathrm{d} \Pi(q)}{\mathrm{d} s}=\mathrm{d} \Pi .\left(X+u_{1} Y_{1}\right) \psi^{\prime}
$$

où $\psi^{\prime}=\frac{v}{r} \cos \gamma$, que l'on note $(\tilde{X}, \tilde{Y})$. L'algèbre de Lie $\mathcal{L}_{\Pi}$ engendrée par $\mathrm{d} \Pi\left(X, Y_{1}\right)$ est de rang 3 d'après la section 4.4. Il en est de même de l'algèbre de Lie $\tilde{\mathcal{L}}$ engendrée par $(\tilde{X}, \tilde{Y})$. Notons $\tilde{\mathcal{L}}_{0}$ l'idéal de $\tilde{\mathcal{L}}$ (de codimension 0 ou 1) engendré par $\left\{a d^{k} \tilde{X} . \tilde{Y}, k=0 \ldots+\infty\right\}$. L'ensemble des états accessibles $R\left(\Pi\left(q_{0}\right), s\right)$ où $s$ est un paramètre fixé est lié aux feuilles intégrales de $\tilde{\mathcal{L}}_{0}$. Le paramètre $s$ est donné par les conditions initiales $\chi(0), L(0)$. Le raisonnement est valide tant que $\cos \chi \neq 0$. 
Contrôle $\mathbf{u}_{\mathbf{1}}=\mathbf{0}$. Le contrôle $u_{2}$ permet de contrôler la dynamique dans le plan $(\chi, L)$. En paramétrant par $L$ le système s'écrit après feedback :

$$
\frac{\mathrm{d} \chi}{\mathrm{d} L}=\tan \chi \tan L+v_{2}, v_{2}=\frac{u_{2} k^{\prime} \rho v}{\cos ^{2} \gamma}
$$

Le rôle de $v_{2}$ est donc de transférer le système de $\chi(L(0))$ à une valeur finale $\chi\left(\frac{\pi}{2}\right)$ prescrite. Cela permet de découpler le problème de contrôle.

Découplage : $\chi \neq \pm \frac{\pi}{2}$

- Calculer $u_{2}$ pour transférer $\chi(L(0))$ à la valeur finale $\chi_{d}$ en un temps $L=\frac{\pi}{2}$.

- Avec d $s s=\int_{L(0)}^{\frac{\pi}{2}} \frac{\mathrm{d} L}{\cos \chi(L)}$, calculer $u_{1}$ pour transférer (avec le système $\left.(\tilde{X}, \tilde{Y})\right) \Pi(q)=(r, v, \gamma)$ de sa position initiale à sa position finale en un temps $s$.

\subsubsection{Analyse des extrémales pour le problème convexifié}

Hypothèse. Pour cette étude on se place en dehors du point cible $L= \pm \frac{\pi}{2}$ où le système n'est pas analytique du fait du choix de la carte pour le représenter.

Extrémales singulières. On calcule les extrémales singulières pour le système :

$$
\frac{\mathrm{d} q}{\mathrm{~d} t}=\bar{X}(q)+u_{1} \bar{Y}_{1}(q)+u_{2} \bar{Y}_{2}(q) .
$$

Les arcs singuliers pour le problème convexifié doivent satisfaire la contrainte : $u_{1}^{2}+u_{2}^{2} \leq 1$, et pour le problème initial : $u_{1}^{2}+u_{2}^{2}=1$. Ce sont aussi des arcs singuliers pour le système mono-entrée où le contrôle est $v=\dot{\mu}$, dérivée de la gîte.

Par invariance par feedback, les extrémales singulières sont celles du système :

$$
\frac{\mathrm{d} q}{\mathrm{~d} t}=\bar{X}+\tilde{u}_{1} Y_{1}+\tilde{u}_{2} Y_{2}
$$

avec $\bar{X}=\psi X, \psi=\frac{1}{\varphi}=f(\rho, v), \varphi=C_{q} \sqrt{\rho} v^{3}$, et :

$$
X=v \sin \gamma \frac{\partial}{\partial r}-\left(g \sin \gamma+k \rho v^{2}\right) \frac{\partial}{\partial v}+\frac{v}{r} \cos \gamma \cos \chi \frac{\partial}{\partial L}, \quad Y_{1}=\frac{\partial}{\partial \gamma}, \quad Y_{2}=\frac{\partial}{\partial \chi} .
$$

En particulier on remarque que $Y_{1}$ et $Y_{2}$ commutent, et que $Y_{1} . \psi=Y_{2} . \psi=0$. Posons $p=\left(p_{r}, p_{v}, p_{\gamma}, p_{\chi}, p_{L}\right)$. Les extrémales singulières sont par définition contenues dans la surface $\Sigma$ de commutation : $\left\langle p, Y_{1}\right\rangle=\left\langle p, Y_{2}\right\rangle=0$, soit :

$$
p_{\gamma}=p_{\chi}=0
$$

En dérivant par rapport à $t$ on obtient, avec $\left[Y_{1}, Y_{2}\right]=0$, les contraintes :

$$
\left\langle p,\left[X, Y_{1}\right]\right\rangle=\left\langle p,\left[X, Y_{2}\right]\right\rangle=0 .
$$

Or :

$$
\left[X, Y_{1}\right]=-v \cos \gamma \frac{\partial}{\partial r}+g \cos \gamma \frac{\partial}{\partial v}+\frac{v}{r} \sin \gamma \cos \chi \frac{\partial}{\partial L}, \quad\left[X, Y_{2}\right]=\frac{v}{r} \cos \gamma \sin \chi \frac{\partial}{\partial L}
$$


ce qui nous conduit à définir $\Sigma^{\prime}$ par les équations :

$$
\begin{aligned}
-v \cos \gamma p_{r}+g \cos \gamma p_{v}+\frac{v}{r} \sin \gamma \cos \chi p_{L} & =0 \\
p_{L} \frac{v}{r} \cos \gamma \sin \chi & =0 .
\end{aligned}
$$

En redérivant les contraintes on obtient :

$$
\begin{aligned}
\left\langle p,\left[\bar{X},\left[\bar{X}, Y_{1}\right]\right]+\tilde{u}_{1}\left[Y_{1},\left[\bar{X}, Y_{1}\right]\right]+\tilde{u}_{2}\left[Y_{2},\left[\bar{X}, Y_{1}\right]\right]\right\rangle=0 \\
\left\langle p,\left[\bar{X},\left[\bar{X}, Y_{2}\right]\right]+\tilde{u}_{1}\left[Y_{1},\left[\bar{X}, Y_{2}\right]\right]+\tilde{u}_{2}\left[Y_{2},\left[\bar{X}, Y_{2}\right]\right]\right\rangle=0 .
\end{aligned}
$$

Il faut donc calculer les crochets de Lie de longueur 3. On a :

$$
\left\langle p,\left[Y_{1},\left[\bar{X}, Y_{1}\right]\right]\right\rangle=\left\langle p,\left[Y_{1}, \psi\left[X, Y_{1}\right]\right]\right\rangle=\psi\left\langle p,\left[Y_{1},\left[X, Y_{1}\right]\right]\right\rangle
$$

et de même :

$$
\begin{aligned}
\left\langle p,\left[Y_{2},\left[\bar{X}, Y_{1}\right]\right]\right\rangle & =\psi\left\langle p,\left[Y_{2},\left[X, Y_{1}\right]\right]\right\rangle \\
\left\langle p,\left[Y_{1},\left[\bar{X}, Y_{2}\right]\right]\right\rangle & =\psi\left\langle p,\left[Y_{1},\left[X, Y_{2}\right]\right]\right\rangle \\
\left\langle p,\left[Y_{2},\left[\bar{X}, Y_{2}\right]\right]\right\rangle & =\psi\left\langle p,\left[Y_{2},\left[X, Y_{2}\right]\right]\right\rangle \cdot
\end{aligned}
$$

On obtient après calculs :

$$
\begin{aligned}
& {\left[Y_{1},\left[X, Y_{1}\right]\right]=v \sin \gamma \frac{\partial}{\partial r}-g \sin \gamma \frac{\partial}{\partial v}+\frac{v}{r} \cos \gamma \cos \chi \frac{\partial}{\partial L}} \\
& {\left[Y_{2},\left[X, Y_{1}\right]\right]=-\frac{v}{r} \sin \gamma \sin \chi \frac{\partial}{\partial L}} \\
& {\left[Y_{1},\left[X, Y_{2}\right]\right]=-\frac{v}{r} \sin \gamma \sin \chi \frac{\partial}{\partial L}} \\
& {\left[Y_{2},\left[X, Y_{2}\right]\right]=\frac{v}{r} \cos \gamma \cos \chi \frac{\partial}{\partial L} .}
\end{aligned}
$$

On peut résumer ces calculs en :

\section{Lemme 4.18.}

1. La surface $\Sigma^{\prime}$ se stratifie en :

(a) $\Sigma_{1}^{\prime}: p_{L}=0,-v \cos \gamma p_{r}+g \cos \gamma p_{v}=0$;

(b) $\Sigma_{2}^{\prime}: \chi=k \pi,-v \cos \gamma p_{r}+g \cos \gamma p_{v}+\frac{v}{r} \sin \gamma \varepsilon p_{L}=0$ ò̀ $\varepsilon=\cos \chi= \pm 1$.

2. $\left[Y_{1},\left[X, Y_{1}\right]\right]=X+k \rho v^{2} \frac{\partial}{\partial v}$.

De l'étude de la section 4.4, on en déduit le résultat :

Corollaire 4.19. Si $p_{L}=0$ il n'existe pas d'extrémales singulières.

Démonstration. La surface $p_{L}=p_{\chi}=0$ est invariante par le flot extrémal, et l'analyse des extrémales se ramène à l'analyse du sous-système I. Or d'après la section 4.4.2 il n'existe pas d'extrémale singulière pour le système I.

Le cas $\sin \chi=\mathbf{0}$. On a alors $\left[Y_{2},\left[X, Y_{1}\right]\right]=\left[Y_{1},\left[X, Y_{2}\right]\right]=0$ et $\left[Y_{2},\left[X, Y_{2}\right]\right]=\frac{v}{r} \cos \gamma \cos k \pi$. On va alors calculer $\tilde{u}_{2}$ en utilisant (32). En fait un calcul direct donne le résultat. En effet :

$$
\dot{\chi}=\frac{v}{r} \cos \gamma \tan L \sin \chi+u_{2} \frac{k^{\prime} v}{\cos \gamma} .
$$


Imposant $\chi=k \pi$ on doit donc avoir $\dot{\chi}=0$, et donc $u_{2}=0$. Ceci implique donc la condition $u_{1}^{2} \leq 1$ pour le problème convexifié et $u_{1}^{2}=1$ pour le problème initial.

On se ramène alors à considérer le problème réduit mono-entrée :

$$
\dot{q}=\bar{X}_{r}+\tilde{u} Y
$$

avec $\bar{X}_{r}=\psi X_{r}, X_{r}=v \sin \gamma \frac{\partial}{\partial r}-\left(g \sin \gamma+k \rho v^{2}\right) \frac{\partial}{\partial v}+\left(\frac{v}{r} \cos \gamma \varepsilon\right) \frac{\partial}{\partial L}, \varepsilon= \pm 1, Y=\frac{\partial}{\partial \gamma}$.

Lemme 4.20. Les extrémales singulières sont localisées en $\chi=k \pi$ et sont celles du système réduit (33).

On note $\tilde{u}_{s}$ le contrôle singulier après feedback. Les détails des calculs sont les suivants :

$$
\begin{aligned}
\langle p, Y\rangle=\left\langle p,\left[\bar{X}_{r}, Y\right]\right\rangle & =0 \\
\left\langle p,\left[\bar{X}_{r},\left[\bar{X}_{r}, Y\right]\right]+\tilde{u}_{s}\left[Y,\left[\bar{X}_{r}, Y\right]\right]\right\rangle & =0
\end{aligned}
$$

et

$$
\begin{aligned}
{\left[\bar{X}_{r}, Y\right] } & =\psi\left[\bar{X}_{r}, Y\right] \\
{\left[Y,\left[\bar{X}_{r}, Y\right]\right] } & =\psi\left[Y,\left[\bar{X}_{r}, Y\right]\right] \\
{\left[\bar{X}_{r},\left[\bar{X}_{r}, Y\right]\right] } & =\psi^{2}\left[X_{r},\left[X_{r}, Y\right]\right]+\psi\left(X_{r} . \psi\right)\left[X_{r}, Y\right]-\psi\left(\left[X_{r}, Y\right] . \psi\right) X
\end{aligned}
$$

puis :

$$
\left[X_{r},\left[X_{r}, Y\right]\right]=\cos \gamma k \rho v^{2} \frac{\partial}{\partial r}+\left(2 k \rho v g \cos \gamma-k \rho^{\prime} v^{3} \cos \gamma\right) \frac{\partial}{\partial v}-\left(\frac{v^{2}}{r^{2}} \varepsilon+\frac{g}{r} \varepsilon+\frac{k \rho v^{2}}{r} \sin \gamma \varepsilon\right) \frac{\partial}{\partial L} .
$$

Le contrôle singulier est donné par :

$$
\langle p, Y\rangle=\langle p,[X, Y]\rangle=0
$$

et

$$
\tilde{u}_{s}=-\frac{\left\langle p,\left[\bar{X}_{r},\left[\bar{X}_{r}, Y\right]\right]\right\rangle}{\left\langle p,\left[Y,\left[\bar{X}_{r}, Y\right]\right]\right\rangle}
$$

soit après calcul :

$$
\tilde{u}_{s}=-\frac{1}{2} \frac{\left(\beta k \cos \gamma \rho r^{2} v^{4} p_{r}-\beta k \sin \gamma \rho r v^{4} \varepsilon p_{L}-2 g v^{2} \varepsilon p_{L}+g \beta r v^{2} \varepsilon p_{L}+4 g^{2} r \varepsilon p_{L}\right) \cos \gamma}{C_{q} g r \sqrt{\rho} v^{4} \varepsilon p_{L}} .
$$

Il dépend du paramètre $p_{L}$. Et, en utilisant la relation :

$$
\dot{\gamma}=\psi \cos \gamma\left(-\frac{g}{v}+\frac{v}{r}\right)+\psi k^{\prime} \rho v u_{1}
$$

et l'expression (36) on peut calculer le contrôle singulier $u_{1 s}$ :

$$
u_{1 s}=\alpha+\beta \tilde{u}_{s}=-\frac{\cos \gamma}{k^{\prime} \rho v}\left(-\frac{g}{v}+\frac{v}{r}\right)+\frac{\tilde{u}_{s}}{\psi k^{\prime} \rho v} .
$$

D'où le test d'admissibilité $\left|u_{1 s}\right| \leq 1$ pour le problème convexifié et $\left|u_{1 s}\right|=1$ pour le problème original.

Optimalité. On considère le système mono-entrée :

$$
\dot{q}=\bar{X}_{r}+u Y
$$


où la contrainte sur $u$ est relaxée : $u \in \mathbb{R}$. Pour tester l'optimalité pour le problème du temps optimal on utilise :

1. la condition nécessaire de Legendre-Clebsch;

2. les conditions suffisantes de [5] établies sous des conditions génériques.

Condition nécessaire de Legendre-Clebsch. Elle s'écrit :

$$
\begin{array}{r}
\left\langle p, \bar{X}_{r}\right\rangle\left\langle p,\left[Y,\left[\bar{X}_{r}, Y\right]\right]\right\rangle \geq 0 \\
\operatorname{avec}\langle p, Y\rangle=\left\langle p,\left[\bar{X}_{r}, Y\right]\right\rangle=0 .
\end{array}
$$

On obtient donc avec $\psi>0$ :

$$
p_{\gamma}=0 \text { et }-v \cos \gamma p_{r}+g \cos \gamma p_{v}+\frac{v}{r} \sin \gamma \varepsilon p_{L}=0
$$

soit donc :

$$
p_{\gamma}=0 \text { et } p_{v}=\frac{v}{g} p_{r}-\frac{v}{r g} \tan \gamma \varepsilon p_{L}
$$

et comme $\psi>0$, on doit donc tester :

$$
\left\langle p, X_{r}\right\rangle\left\langle p,\left[Y,\left[X_{r}, Y\right]\right]\right\rangle \geq 0 .
$$

Or d'après le lemme 4.18 :

Donc avec (39) :

$$
\left[Y,\left[X_{r}, Y\right]\right]=X_{r}+k \rho v^{2} \frac{\partial}{\partial v}
$$

$$
\begin{gathered}
\left\langle p, X_{r}\right\rangle=\frac{v}{r \cos \gamma} \varepsilon p_{L}+\frac{k}{g r} \rho v^{3} \tan \gamma \varepsilon p_{L}-\frac{k}{g} \rho v^{3} p_{r} \\
\left\langle p,\left[Y,\left[X_{r}, Y\right]\right]\right\rangle=\langle p, X\rangle+k \rho v^{2} p_{v}=\frac{v}{r \cos \gamma} \varepsilon p_{L} .
\end{gathered}
$$

D'où :

Lemme 4.21. Puisque $\cos \gamma \neq 0$ dans le domaine de vol, on a :

$$
\text { Signe }\left\langle p, \bar{X}_{r}\right\rangle\left\langle p,\left[Y,\left[\bar{X}_{r}, Y\right]\right]\right\rangle=\text { Signe }\left(1+\frac{k}{g} \rho v^{2} \sin \gamma-\frac{k}{g} \rho v^{2} r \cos \gamma \varepsilon \frac{p_{r}}{p_{L}}\right) .
$$

Conditions suffisantes de [5]. On les rappelle brièvement dans le cas d'un système de dimension 4. Notons :

$$
E_{1}=Y, E_{2}=\left[\bar{X}_{r}, Y\right], E_{3}=\left[\bar{X}_{r},\left[\bar{X}_{r}, Y\right]\right]+\tilde{u}_{s}\left[Y,\left[\bar{X}_{r}, Y\right]\right] \text { et } E=\operatorname{Vect}\left\{E_{1}, E_{2}, E_{3}\right\} .
$$

On fait les hypothèses suivantes :

- $\operatorname{dim} E=\mathbb{R}^{3}$;

- $\left[Y,\left[\bar{X}_{r}, Y\right]\right] \notin E$.

Si $\bar{X}_{r} \in E$ on est dans le cas dit exceptionnel : $H=\left\langle p, \bar{X}_{r}+\tilde{u}_{s} Y\right\rangle=0$, et on suppose de plus :

- $\operatorname{dim} \operatorname{Vect}\left\{E_{1}, E_{2}, \bar{X}_{r}\right\}=3$.

Sinon, on définit la fonction $a(q)$ par :

$$
\left[Y,\left[\bar{X}_{r}, Y\right]\right]=a \bar{X}_{r} \quad \bmod E .
$$


On a d'après [5] la proposition suivante :

Proposition 4.22. Sous les hypothèses précédentes on a:

1. dans le cas exceptionnel, la trajectoire singulière est $C^{0}$-temps-minimale pour des temps assez petits ( $t<$ $\left.t_{c c}\right)$;

2. si $a>0$ on est dans la situation dite hyperbolique, et la trajectoire est $C^{0}$-temps-minimale pour des temps assez petits $\left(t<t_{c}\right)$;

3. si $a<0$, on est dans la situation dite elliptique, et la trajectoire est $C^{0}$-temps-maximale pour des temps assez petits $\left(t<t_{c}\right)$.

De plus les temps $t_{c}, t_{c c}$ sont appelés temps conjugués et peuvent être calculés.

Dans notre cas, on a :

\section{Lemme 4.23.}

1. Les trois vecteurs $E_{1}, E_{2}, E_{3}$ sont indépendants.

2. Les trois vecteurs $E_{1}, E_{2}, \bar{X}_{r}$ sont indépendants.

3. $a=\frac{g \varepsilon p_{L}}{g \varepsilon p_{L}-k \rho r v^{2} \cos \gamma p_{r}+k \rho v^{2} \sin \gamma \varepsilon p_{L}}$.

4. Le cas exceptionnel correspond $\grave{a}$ :

$$
\begin{aligned}
p_{r} & =\left(\frac{g}{v}+k \rho v \sin \gamma\right) p_{v} \\
\varepsilon p_{L} & =k \rho r v \cos \gamma p_{v}
\end{aligned}
$$

et le contrôle singulier dans le cas exceptionnel se simplifie en:

$$
\tilde{u}_{s}=\frac{\cos \gamma}{C_{q} \sqrt{\rho} r v^{4}}\left(-\beta r v^{2}+v^{2}-2 g r\right) .
$$

Valeurs numériques. Les extrémales exceptionnelles $(H=0)$ sont critiques et séparent la zone hyperbolique de la zone elliptique, voir figure 8.

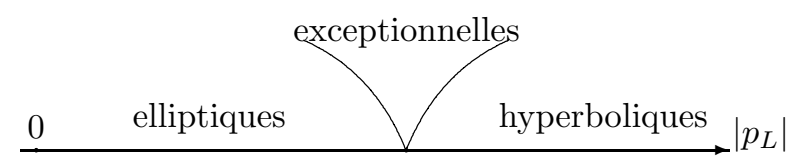

FiguRE 8

On peut se donner une idée des valeurs numériques en considérant des points significatifs du vol. Les valeurs constantes sont les suivantes :

$$
\begin{aligned}
& \rho(r)=\rho_{0} \mathrm{e}^{-\beta r}=\bar{\rho}_{0} \mathrm{e}^{-\frac{r-r_{0}}{h_{s}}} \quad \text { où } \bar{\rho}_{0}=1,22499 \mathrm{~kg} / \mathrm{m}^{3}, h_{s}=7143 \mathrm{~m}, r_{0}=6378140 \mathrm{~m} \\
& k=\frac{S C_{D}}{2 m} \quad \text { où } S=100 \mathrm{~m}^{2}, C_{D}=0.5, m=7169,6 \mathrm{~kg}, \text { donc } k \simeq 3,510^{-3} \text { USI } \\
& k^{\prime}=\frac{S C_{L}}{2 m} \quad \text { où } C_{L}=0,1, \text { donc } k^{\prime} \simeq 710^{-4} \text { USI } \\
& C_{q}=1,70510^{-5} \text { USI, } g=9,78033 \mathrm{~m} / \mathrm{s}^{2} .
\end{aligned}
$$


Avec ces valeurs numériques, estimons les quantités correspondant aux extrémales exceptionnelles. Considérons tout d'abord les données suivantes (qui correspondent à des valeurs initiales du vol) :

$$
r \simeq 6497960 \mathrm{~m}, v \simeq 710^{3} \mathrm{~m} / \mathrm{s}, \gamma \simeq-1 \text { degrés. }
$$

On en déduit que $\rho(r) \simeq 6,310^{-8} \mathrm{~kg} / \mathrm{m}^{3}$. D'autre part, le vecteur adjoint en un point étant défini à un scalaire près, on peut supposer que $p_{v}=1$. On obtient alors d'après le lemme 4.23 :

$$
p_{v}=1, p_{r} \simeq 1,410^{-3}, \varepsilon p_{L} \simeq 10, \tilde{u}_{s} \simeq-6,610^{-4} .
$$

Le contrôle singulier exceptionnel $\left(u_{1 s}, u_{2 s}\right)$ original (avant feedback) vaut alors, d'après la formule (37) :

$$
u_{1 s} \simeq-310^{6}, u_{2 s}=0
$$

et donc il n'est pas admissible en début de trajectoire.

Considérons maintenant les données suivantes, en fin de trajectoire :

$$
r \simeq 6394860 \mathrm{~m}, v \simeq 443,52 \mathrm{~m} / \mathrm{s}, \gamma=-15,2 \text { degrés. }
$$

Par contre les valeurs précédentes de $C_{D}, C_{L}$ ne peuvent plus être valables, car ces valeurs dépendent du nombre de Machs. On prend donc les estimations suivantes (d'après le cahier des charges du CNES) $: C_{D}=C_{L}=0,25$. Ceci change les valeurs de $k, k^{\prime}$. On obtient alors pour le contrôle singulier exceptionnel :

$$
u_{1 s} \simeq-0,89, u_{2 s}=0
$$

Autrement dit à basse altitude (environ $20 \mathrm{~km}$ ), et pour des vitesses de l'ordre de $500 \mathrm{~m} / \mathrm{s}$, le contrôle singulier exceptionnel est admissible pour le problème convexifié $u_{1}^{2}+u_{2}^{2} \leq 1$. Par conséquent il existe aussi, pour le problème convexifié, des trajectoires elliptiques et hyperboliques admissibles.

Vérifions d'autre part que ce contrôle singulier exceptionnel admissible satisfait les contraintes sur l'état. Les données numériques sont les suivantes :

- contrainte sur le flux thermique : $\varphi=C_{q} \sqrt{\rho} v^{3} \leq \varphi^{\max }$, où $\varphi^{\max }=717300 \mathrm{~W} / \mathrm{m}^{2}$. Avec les valeurs précédentes :

$$
\varphi \simeq 510 \mathrm{~W} / \mathrm{m}^{2}
$$

- contrainte sur la pression dynamique : $P=\frac{1}{2} \rho v^{2} \leq P^{\max }$, où $P^{\max }=22220$ Pa. Ici on a :

$$
P \simeq 11600 \mathrm{~Pa}
$$

Les contraintes sur l'état sont donc satisfaites.

Décrivons maintenant grossièrement l'allure de cet arc exceptionnel. Avec ces données numériques, on a $|g \sin \gamma| \ll k \rho v^{2}$, i.e. la pesanteur est négligeable par rapport à la force de traînée. D'autre part : $\dot{\gamma} \simeq 310^{-6}$. Donc si au début de l'arc singulier $\gamma$ est de l'ordre du degré, alors on peut considérer que $\gamma$ est constante le long de l'arc (ce qui est réaliste, puisque de toute façon la durée totale de l'arc atmosphérique n'excède pas $3000 \mathrm{~s}$ ). Des simulations numériques plus précises doivent cependant être conduites. Ce calcul suggère en tout cas qu'en fin de trajectoire il existe des arcs singuliers admissibles (elliptiques, hyperboliques et exceptionnels).

Analyse des extrémales régulières. Le contrôle extrémal prend alors presque partout ses valeurs sur le bord du domaine $u_{1}^{2}+u_{2}^{2} \leq 1$. Le Hamiltonien s'écrit :

$$
H=\left\langle p, \bar{X}+u_{1} \bar{Y}_{1}+u_{2} \bar{Y}_{2}\right\rangle
$$


avec $\bar{X}=\psi X, \bar{Y}_{1}=\psi Y_{1}, \bar{Y}_{2}=\psi Y_{2}$, et :

$$
\begin{aligned}
X & =v \sin \gamma \frac{\partial}{\partial r}-\left(g \sin \gamma+k \rho v^{2}\right) \frac{\partial}{\partial v}+\cos \gamma\left(-\frac{g}{v}+\frac{v}{r}\right) \frac{\partial}{\partial \gamma}+\frac{v}{r} \cos \gamma \sin \chi \tan L \frac{\partial}{\partial \chi}+\frac{v}{r} \cos \gamma \cos \chi \frac{\partial}{\partial L} \\
Y_{1} & =k^{\prime} \rho v \frac{\partial}{\partial \gamma}, Y_{2}=\frac{k^{\prime} \rho v}{\cos \gamma} \frac{\partial}{\partial \chi} .
\end{aligned}
$$

Notons $\phi_{i}=\left\langle p, \bar{Y}_{i}\right\rangle, i=1,2$ et soit $\phi=\left(\phi_{1}, \phi_{2}\right)$ la fonction de commutation. D'après le Principe du Minimum on doit minimiser $H$ sous la contrainte $u_{1}^{2}+u_{2}^{2} \leq 1$. Notons $z_{0}=\left(q_{0}, p_{0}\right), p_{0} \neq 0$.

Points d'ordre 0. Si $\|\phi\| \neq 0$ en $z_{0}$, le point $z_{0}$ est dit d'ordre 0 , et le contrôle extrémal est donné par :

$$
u=-\frac{\left(\phi_{1}, \phi_{2}\right)}{\|\phi\|}
$$

Ici : $\phi_{1}=\psi k^{\prime} \rho v p_{\gamma}$ et $\phi_{2}=\psi \frac{k^{\prime} \rho v}{\cos \gamma} p_{\chi}$, donc :

$$
\|\phi\|=\psi \frac{k^{\prime} \rho v}{\cos \gamma} \sqrt{p_{\chi}^{2}+\cos ^{2} \gamma p_{\gamma}^{2}}, \quad u_{1}=\frac{-\cos \gamma p_{\gamma}}{\sqrt{p_{\chi}^{2}+\cos ^{2} \gamma p_{\gamma}^{2}}}, \quad u_{2}=\frac{-p_{\chi}}{\sqrt{p_{\chi}^{2}+\cos ^{2} \gamma p_{\gamma}^{2}}} .
$$

Pour analyser les extrémales telles que $\|\phi\| \neq 0$ on doit considérer l'équation :

$$
\begin{aligned}
& \dot{q}=\bar{X}+u_{1} \bar{Y}_{1}+u_{2} \bar{Y}_{2} \\
& \dot{p}=-p\left(\frac{\partial \bar{X}}{\partial q}+u_{1} \frac{\partial \bar{Y}_{1}}{\partial q}+u_{2} \frac{\partial \bar{Y}_{2}}{\partial q}\right)
\end{aligned}
$$

qui n'est pas Hamiltonienne en général du fait du feedback extrémal $u$. On régularise cette équation en changeant le paramétrage en $d s=\frac{d t}{\|\phi\|}$. Ici on va plutôt poser :

$$
\mathrm{d} s=\frac{\mathrm{d} t}{\sqrt{p_{\chi}^{2}+\cos ^{2} \gamma p_{\gamma}^{2}}} .
$$

Notons ' la dérivée par rapport à $s$. Dans ce nouveau paramétrage, le système Hamiltonien (43) s'écrit :

$$
\begin{aligned}
r^{\prime} & =\psi v \sin \gamma \sqrt{p_{\chi}^{2}+\cos ^{2} \gamma p_{\gamma}^{2}} \\
v^{\prime} & =-\psi\left(g \sin \gamma+k \rho v^{2}\right) \sqrt{p_{\chi}^{2}+\cos ^{2} \gamma p_{\gamma}^{2}} \\
\gamma^{\prime} & =\psi \cos \gamma\left(-\frac{g}{v}+\frac{v}{r}\right) \sqrt{p_{\chi}^{2}+\cos ^{2} \gamma p_{\gamma}^{2}}-\psi k^{\prime} \rho v \cos \gamma p_{\gamma} \\
\chi^{\prime} & =\psi \frac{v}{r} \cos \gamma \sin \chi \tan L \sqrt{p_{\chi}^{2}+\cos ^{2} \gamma p_{\gamma}^{2}}-\psi \frac{k^{\prime} \rho v}{\cos \gamma} p_{\chi} \\
L^{\prime} & =\psi \frac{v}{r} \cos \gamma \cos \chi \sqrt{p_{\chi}^{2}+\cos ^{2} \gamma p_{\gamma}^{2}}
\end{aligned}
$$




$$
\begin{aligned}
& p_{r}^{\prime}=\left(-\frac{\partial \psi}{\partial r} v \sin \gamma p_{r}+\left(\frac{\partial \psi}{\partial r}\left(g \sin \gamma+k \rho v^{2}\right)+\psi k \frac{\partial \rho}{\partial r} v^{2}\right) p_{v}+\left(-\frac{\partial \psi}{\partial r} \cos \gamma\left(-\frac{g}{v}+\frac{v}{r}\right)+\psi \frac{v}{r^{2}} \cos \gamma\right) p_{\gamma}\right. \\
& \left.+\left(-\frac{\partial \psi}{\partial r} \frac{v}{r} \cos \gamma \sin \chi \tan L+\psi \frac{v}{r^{2}} \cos \gamma \sin \chi \tan L\right) p_{\chi}+\left(-\frac{\partial \psi}{\partial r} \frac{v}{r} \cos \gamma \cos \chi+\psi \frac{v}{r^{2}} \cos \gamma \cos \chi\right) p_{L}\right) \\
& \times \sqrt{p_{\chi}^{2}+\cos ^{2} \gamma p_{\gamma}^{2}}+\left(\frac{\partial \psi}{\partial r} \rho+\psi \frac{\partial \rho}{\partial r}\right) \frac{k^{\prime} v}{\cos \gamma}\left(p_{\chi}^{2}+\cos ^{2} \gamma p_{\gamma}^{2}\right) \\
& p_{v}^{\prime}=\left(-\left(\psi+\frac{\partial \psi}{\partial v} v\right) \sin \gamma p_{r}+\left(\frac{\partial \psi}{\partial v}\left(g \sin \gamma+k \rho v^{2}\right)+2 \psi k \rho v\right) p_{v}\right. \\
& +\left(-\frac{\partial \psi}{\partial v} \cos \gamma\left(-\frac{g}{v}+\frac{v}{r}\right)-\psi \cos \gamma\left(\frac{g}{v^{2}}+\frac{1}{r}\right)\right) p_{\gamma} \\
& -\left(\frac{\partial \psi}{\partial v} \frac{v}{r} \cos \gamma \sin \chi \tan L+\frac{\psi}{r} \cos \gamma \sin \chi \tan L\right) p_{\chi} \\
& \left.-\left(\psi+\frac{\partial \psi}{\partial v} v\right) \frac{1}{r} \cos \gamma \cos \chi p_{L}\right) \sqrt{p_{\chi}^{2}+\cos ^{2} \gamma p_{\gamma}^{2}} \\
& +\left(\psi+\frac{\partial \psi}{\partial v} v\right) \frac{k^{\prime} \rho}{\cos \gamma}\left(p_{\chi}^{2}+\cos ^{2} \gamma p_{\gamma}^{2}\right) \\
& p_{\gamma}^{\prime}=\psi\left(-v \cos \gamma p_{r}+g \cos \gamma p_{v}+\left(-\frac{g}{v}+\frac{v}{r}\right) \sin \gamma p_{\gamma}+\frac{v}{r} \sin \gamma \sin \chi \tan L p_{\chi}+\frac{v}{r} \sin \gamma \cos \chi p_{L}\right) \\
& \times \sqrt{p_{\chi}^{2}+\cos ^{2} \gamma p_{\gamma}^{2}}+\psi \frac{k^{\prime} \rho v}{\cos \gamma} \tan \gamma p_{\chi}^{2} \\
& p_{\chi}^{\prime}=\psi \frac{v}{r} \cos \gamma\left(-\cos \chi \tan L p_{\chi}+\sin \chi p_{L}\right) \sqrt{p_{\chi}^{2}+\cos ^{2} \gamma p_{\gamma}^{2}} \\
& p_{L}^{\prime}=-\psi \frac{v}{r} \frac{\cos \gamma \sin \chi}{\cos ^{2} L} p_{\chi} \sqrt{p_{\chi}^{2}+\cos ^{2} \gamma p_{\gamma}^{2}}
\end{aligned}
$$

En un point d'ordre 0, ce système (45) est lisse et redressable. Pour classifier les extrémales on va donc s'intéresser aux points singuliers de ce système localisés dans $\phi=0$.

Le cadre géométrique. La classification des extrémales au voisinage de $\phi=0$ est un problème d'analyse des solutions d'une équation différentielle $\frac{\mathrm{d} z}{\mathrm{~d} s}=V(z)$ au voisinage de points singuliers (contenus dans $\phi=0$ ). Une difficulté technique majeure est que ces points singuliers ne sont pas isolés et que le théorème d'HartmanGrobman d'équivalence topologique avec le système linéarisé ne s'applique pas car les points singuliers ne sont pas hyperboliques. La construction d'un modèle local au voisinage de chaque singularité est un problème difficile et pose le problème d'approcher le système par des modèles dont il n'est pas clair que l'on puisse les choisir nilpotents. En particulier ce travail est la généralisation au cas multi-entrées des travaux de [14] sur la classification des extrémales au voisinage de la surface de commutation dans le cas mono-entrée.

Points d'ordre $\geq 1$. La surface $\phi=0$ correspond à la surface de commutation :

$$
\left\langle p, \bar{Y}_{1}(q)\right\rangle=\left\langle p, \bar{Y}_{2}(q)\right\rangle=0
$$

qui est la surface $\Sigma: p_{\gamma}=p_{\chi}=0$. En dérivant par rapport à $t$ il vient :

$$
\begin{aligned}
\left\langle p,\left[\bar{X}, \bar{Y}_{1}\right](q)+u_{2}\left[\bar{Y}_{2}, \bar{Y}_{1}\right](q)\right\rangle & =0 \\
\left\langle p,\left[\bar{X}, \bar{Y}_{2}\right](q)+u_{1}\left[\bar{Y}_{1}, \bar{Y}_{2}\right](q)\right\rangle & =0 .
\end{aligned}
$$


Or :

$$
\left[\bar{Y}_{2}, \bar{Y}_{1}\right]=\psi^{2}\left[Y_{2}, Y_{1}\right]+\psi\left(Y_{2} \cdot \psi\right) Y_{1}-\psi\left(Y_{1} \cdot \psi\right) Y_{2}=\psi^{2}\left[Y_{2}, Y_{1}\right] \quad \text { car } Y_{1} \cdot \psi=0
$$

Par ailleurs :

$$
\left[Y_{2}, Y_{1}\right]=-\frac{\left(k^{\prime} \rho v\right)^{2} \sin \gamma}{\cos ^{2} \gamma} \frac{\partial}{\partial \chi}
$$

Donc $\left[\bar{Y}_{2}, \bar{Y}_{1}\right]$ est colinéaire à $\bar{Y}_{2}$, et si $\phi=0$ on a aussi $\left\langle p,\left[\bar{Y}_{2}, \bar{Y}_{1}\right]\right\rangle=0$.

Points d'ordre 1. Plaçons-nous en un point singulier du système précédent :

$$
z_{0}=\left(q_{0}, p^{0}\right)=\left(r_{0}, v_{0}, \gamma_{0}, \chi_{0}, L_{0}, p_{r}^{0}, 0,0, p_{L}^{0}\right)
$$

Supposons, sans nuire à la généralité de l'analyse, que $t=0$ en ce point. On a alors $\phi(0)=0$, et : $\dot{\phi}=\left(\dot{\phi}_{1}, \dot{\phi}_{2}\right)$, avec $\dot{\phi}_{i}=\left\langle p^{0},\left[\bar{X}, \bar{Y}_{2}\right]\left(q_{0}\right)\right\rangle$. Donc $t \mapsto \phi(t)$ est $C^{1}$ car $t \mapsto z(t)=(q(t), p(t))$ est $C^{0}$. Si $\dot{\phi}(t) \neq 0$ on dit que $z_{0}$ est un point de commutation d'ordre 1. On peut alors poser :

$$
\phi_{1}(t)=a t+\mathrm{o}(t), \phi_{2}(t)=b t+\mathrm{o}(t) \quad \text { avec } a^{2}+b^{2} \neq 0 .
$$

Par conséquent :

$$
u_{1}(t)=\frac{-a}{\sqrt{a^{2}+b^{2}}} \frac{t}{|t|}+\mathrm{o}(1), u_{2}(t)=\frac{-b}{\sqrt{a^{2}+b^{2}}} \frac{t}{|t|}+\mathrm{o}(1)
$$

et :

$$
p_{\gamma}(t)=\frac{a}{\psi_{0} k^{\prime} \rho_{0} v_{0}} t+\mathrm{o}(t), p_{\chi}(t)=\frac{b \cos \gamma_{0}}{\psi_{0} k^{\prime} \rho_{0} v_{0}} t+\mathrm{o}(t), \sqrt{p_{\chi}^{2}+\cos ^{2} \gamma p_{\gamma}^{2}}=\sqrt{a^{2}+b^{2}} \frac{\cos \gamma_{0}}{\psi_{0} k^{\prime} \rho_{0} v_{0}}|t|+\mathrm{o}(t) .
$$

Au premier ordre, le système (45) admet donc pour modèle au voisinage du point singulier $z_{0}$ le système suivant :

$$
\begin{aligned}
r^{\prime} & =a_{1} \sqrt{p_{\chi}^{2}+\cos ^{2} \gamma_{0} p_{\gamma}^{2}} \\
v^{\prime} & =a_{2} \sqrt{p_{\chi}^{2}+\cos ^{2} \gamma_{0} p_{\gamma}^{2}} \\
\gamma^{\prime} & =a_{3} \sqrt{p_{\chi}^{2}+\cos ^{2} \gamma_{0} p_{\gamma}^{2}}+a_{4} p_{\gamma} \\
\chi^{\prime} & =a_{5} \sqrt{p_{\chi}^{2}+\cos ^{2} \gamma_{0} p_{\gamma}^{2}}+a_{6} p_{\chi} \\
L^{\prime} & =a_{7} \sqrt{p_{\chi}^{2}+\cos ^{2} \gamma_{0} p_{\gamma}^{2}} \\
p_{r}^{\prime} & =a_{8} \sqrt{p_{\chi}^{2}+\cos ^{2} \gamma_{0} p_{\gamma}^{2}} \\
p_{v}^{\prime} & =a_{9} \sqrt{p_{\chi}^{2}+\cos ^{2} \gamma_{0} p_{\gamma}^{2}} \\
p_{\gamma}^{\prime} & =a_{10} \sqrt{p_{\chi}^{2}+\cos ^{2} \gamma_{0} p_{\gamma}^{2}} \\
p_{\chi}^{\prime} & =a_{11} \sqrt{p_{\chi}^{2}+\cos ^{2} \gamma_{0} p_{\gamma}^{2}} \\
p_{L}^{\prime} & =0
\end{aligned}
$$


avec, notamment :

$$
\begin{aligned}
& a_{10}=\psi_{0}\left(-v_{0} \cos \gamma_{0} p_{r}^{0}+g \cos \gamma_{0} p_{v}^{0}+\frac{v_{0}}{r_{0}} \sin \gamma_{0} \cos \chi_{0} p_{L}^{0}\right) \\
& a_{11}=\psi_{0} \frac{v_{0}}{r_{0}} \cos \gamma_{0} \sin \chi_{0} p_{L}^{0}
\end{aligned}
$$

les autres coefficients étant déterminés à l'aide du système (45) en fonction de $z_{0}$.

En remplaçant les expressions de $u_{1}, u_{2}, p_{\gamma}, p_{\chi}$ dans le système (47) (en tenant compte de la paramétrisation en $s$ ) on obtient :

$$
a=a_{10} \psi_{0} k^{\prime} \rho_{0} v_{0}, \quad b=a_{11} \frac{\psi_{0} k^{\prime} \rho_{0} v_{0}}{\cos \gamma_{0}} .
$$

Résumons tout ceci dans la proposition suivante (comparer avec [7]) :

Proposition 4.24. Par tout point d'ordre 1 passe une unique extrémale, associée au contrôle $\left(u_{1}, u_{2}\right)$ donné par la formule (46). Ce contrôle extrémal tourne d'un angle $\pi$ en traversant la surface de commutation $\phi=0$, voir figure 9.

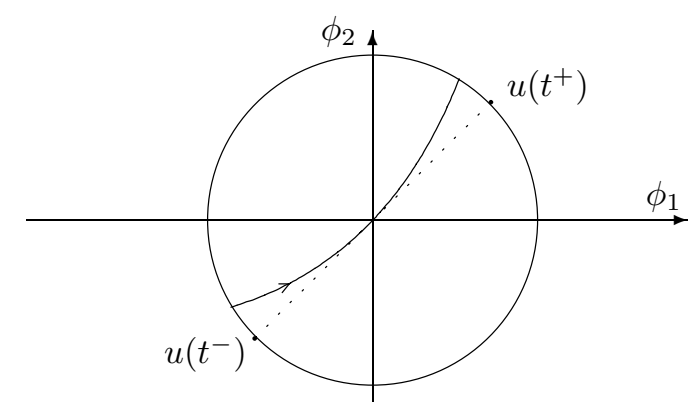

Figure 9

Sur la figure 10 on a représenté l'allure de $p_{\chi}, p_{\gamma}, \gamma$ au point de commutation.
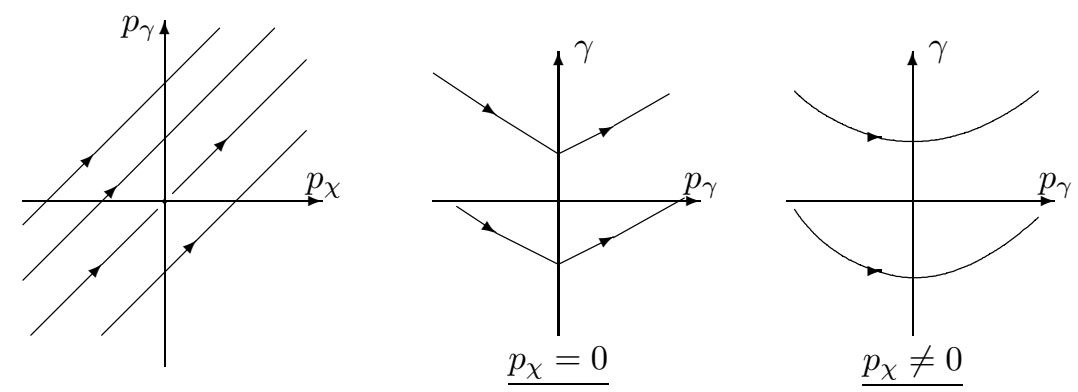

FiguRE 10

Points d'ordre $\geq 2$. Si $\phi=\dot{\phi}=0$, on dit que le point est d'ordre $\geq 2$. Calculons le lieu correspondant. On a :

$$
\begin{aligned}
& {\left[\bar{X}, \bar{Y}_{1}\right]=\psi^{2}\left[X, Y_{1}\right] \quad \bmod \operatorname{Vect}\left\{Y_{1}, Y_{2}\right\}} \\
& {\left[\bar{X}, \bar{Y}_{2}\right]=\psi^{2}\left[X, Y_{2}\right] \quad \bmod \operatorname{Vect}\left\{Y_{1}, Y_{2}\right\}}
\end{aligned}
$$


Or :

$$
\begin{aligned}
& {\left[X, Y_{1}\right]=k^{\prime} \rho v\left(-v \cos \gamma \frac{\partial}{\partial r}+g \cos \gamma \frac{\partial}{\partial v}+\frac{v}{r} \sin \gamma \cos \chi \frac{\partial}{\partial L}\right) \quad \bmod \operatorname{Vect}\left\{Y_{1}, Y_{2}\right\}} \\
& {\left[X, Y_{2}\right]=k^{\prime} \rho \frac{v^{2}}{r} \sin \chi \frac{\partial}{\partial L} \quad \bmod \operatorname{Vect}\left\{Y_{1}, Y_{2}\right\}}
\end{aligned}
$$

Les points d'ordre $\geq 2$ sont donc situés sur la surface $\Sigma \cap \Sigma^{\prime}$ du lemme 4.18, où $\Sigma^{\prime}$ se décompose en deux strates $\Sigma_{1}^{\prime}, \Sigma_{2}^{\prime}$. Traitons les deux strates.

Cas $\chi \neq \mathbf{k} \pi$. Ce cas s'analyse aisément à l'aide de l'analyse du sous-système I. Si $p_{\chi}=p_{L}=0$ en un point alors ils sont identiquement nuls, ainsi que $\phi_{2}$ et $u_{2}$, et donc les extrémales correspondantes sont celles du sous-système I étudié en section 4.4. Un contrôle extrémal est donné par $u_{2}=0$ et $u_{1}=-$ signe $\phi_{1}$, et on a la situation décrite par la figure 11.

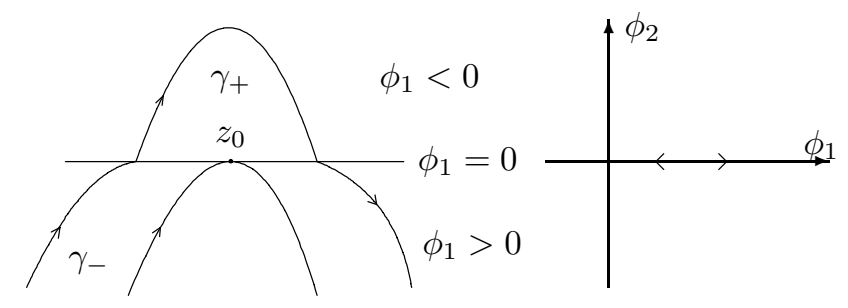

FIGURE 11

Lemme 4.25. Si $\chi \neq k \pi, p_{\chi}=p_{L}=0$ et $-p_{r} v+p_{v} g=0$, on a des points de commutation d'ordre $\geq 2$ ayant les propriétés suivantes:

1. $u_{2}=0$ et $u_{1}=-$ signe $\phi_{1}$ est constant par morceaux;

2. la fonction de commutation est $C^{\infty}$ et possède un point de rebroussement (dégénéré).

Cas $\chi=\mathbf{k} \pi$. Ce cas est plus complexe à analyser. En dérivant $\left\langle p,\left[\bar{X}, \bar{Y}_{i}\right](q)\right\rangle$ il vient :

$$
\begin{aligned}
& \left\langle p,\left[\bar{X},\left[\bar{X}, \bar{Y}_{1}\right]\right](q)+u_{1}\left[\bar{Y}_{1},\left[\bar{X}, \bar{Y}_{1}\right]\right](q)+u_{2}\left[\bar{Y}_{2},\left[\bar{X}, \bar{Y}_{1}\right]\right](q)\right\rangle=0 \\
& \left\langle p,\left[\bar{X},\left[\bar{X}, \bar{Y}_{2}\right]\right](q)+u_{1}\left[\bar{Y}_{1},\left[\bar{X}, \bar{Y}_{2}\right]\right](q)+u_{2}\left[\bar{Y}_{2},\left[\bar{X}, \bar{Y}_{2}\right]\right](q)\right\rangle=0 .
\end{aligned}
$$

Or :

$$
\left[\bar{X},\left[\bar{X}, Y_{2}\right]\right]=\psi^{3}\left[X,\left[X, Y_{2}\right]\right] \bmod \operatorname{Vect}\left\{Y_{i},\left[X, Y_{i}\right]\right\}
$$

et après calcul il vient :

$$
\left[X,\left[X, Y_{2}\right]\right]=0 \bmod \operatorname{Vect}\left\{Y_{i},\left[X, Y_{i}\right]\right\} \text { pour } \chi=k \pi
$$

On a aussi :

$$
\left[\bar{Y}_{1},\left[\bar{X}, \bar{Y}_{2}\right]\right]=\left[\bar{Y}_{2},\left[\bar{X}, \bar{Y}_{1}\right]\right]=0 \bmod \operatorname{Vect}\left\{Y_{i},\left[X, Y_{i}\right]\right\} \text { pour } \chi=k \pi .
$$

En un point d'ordre $\geq 2$ avec $\chi=k \pi$ on a donc :

$$
\phi_{i}(t)=\dot{\phi}_{i}(t)=0, i=1,2
$$


et

$$
\begin{aligned}
& \ddot{\phi}_{1}=\left\langle p,\left[\bar{X},\left[\bar{X}, \bar{Y}_{1}\right]\right](q)+u_{1}\left[\bar{Y}_{1},\left[\bar{X}, \bar{Y}_{1}\right]\right](q)\right\rangle \\
& \ddot{\phi}_{2}=u_{2}\left\langle p,\left[\bar{Y}_{2},\left[\bar{X}, \bar{Y}_{2}\right]\right](q)\right\rangle \cdot
\end{aligned}
$$

En calculant on a :

$$
\left[\bar{Y}_{2},\left[\bar{X}, \bar{Y}_{2}\right]\right]=\psi^{3}\left[Y_{2},\left[X, Y_{2}\right]\right] \quad \bmod \operatorname{Vect}\left\{Y_{i},\left[X, Y_{i}\right]\right\}
$$

et

$$
\left[Y_{2},\left[X, Y_{2}\right]\right]=\left(\frac{k^{\prime} \rho v}{\cos \gamma}\right)^{2} \frac{v}{r} \cos \gamma \cos \chi \frac{\partial}{\partial L} \bmod \operatorname{Vect}\left\{Y_{i},\left[X, Y_{i}\right]\right\}
$$

On a donc au voisinage du point d'ordre $2 z_{0}=\left(q_{0}, p^{0}\right)$ :

$$
\ddot{\phi}_{1}=\alpha+\beta u_{1}, \ddot{\phi}_{2}=\delta u_{2}
$$

où $\alpha=\left\langle p^{0},\left[\bar{X},\left[\bar{X}, \bar{Y}_{1}\right]\right]\left(q_{0}\right)\right\rangle, \beta=\left\langle p^{0},\left[\bar{Y}_{1},\left[\bar{X}, \bar{Y}_{1}\right]\right]\left(q_{0}\right)\right\rangle, \delta=\left\langle p^{0},\left[\bar{Y}_{2},\left[\bar{X}, \bar{Y}_{2}\right]\right]\left(q_{0}\right)\right\rangle$. Par extrémalité on doit avoir en dehors de la surface de commutation :

$$
u_{1}=-\frac{\phi_{1}}{\|\phi\|} \text { et } u_{2}=-\frac{\phi_{2}}{\|\phi\|}
$$

Et donc en reparamétrisant par $\mathrm{d} s=\frac{\mathrm{d} t}{\sqrt{\|\phi\|}}$, le modèle au premier ordre est le suivant :

$$
\begin{aligned}
& \phi_{1}^{\prime \prime}+\beta \phi_{1}=\alpha\|\phi\| \\
& \phi_{2}^{\prime \prime}+\delta \phi_{2}=0
\end{aligned}
$$

et d'après les calculs précédents :

$$
\text { signe } \delta=\text { signe } \beta=\text { signe }\left(\varepsilon p_{L}\right) \text {. }
$$

Par conséquent si $\varepsilon p_{L}>0$ alors $\delta>0$ et donc $\phi_{2}$ oscille. Ce cas doit être analysé plus en détail, et on doit contrôler le nombre d'oscillations par des arguments d'optimalité.

Si $\varepsilon p_{L}<0$ alors $\delta<0$. Soit $\delta_{1}>0$ tel que $\delta_{1}^{2}=-\delta$. Comme $\phi_{2}(s)$ et $\phi_{2}^{\prime}(s)$ doivent tendre vers 0 lorsque $s \rightarrow \infty$, on a :

$$
\phi_{2}(s)=A \mathrm{e}^{-\delta_{1} s}
$$

Estimons $\phi_{1}$. On a l'équation :

$$
\phi_{1}^{\prime \prime}+\beta \phi_{1}=\alpha \sqrt{\phi_{1}^{2}+A^{2} \mathrm{e}^{-2 \delta_{1} s}}
$$

Si $s \rightarrow \infty$ le modèle est donc :

$$
\phi_{1}^{\prime \prime}+\beta \phi_{1}=\alpha\left|\phi_{1}\right|
$$

Le lemme suivant est immédiat :

Lemme 4.26. $\phi_{1}$ oscille si et seulement si $\beta>|\alpha|$.

En particulier si $\phi_{1}$ oscille alors $\beta>0$. Par conséquent on a prouvé :

Lemme 4.27. Si $\varepsilon p_{L}<0$ alors $\phi_{1}$, $\phi_{2}$ n'oscillent pas.

De même que précédemment on a alors : $\phi_{1}(s)=\mathrm{e}^{-\delta_{2} s}$ où $\delta_{2}>0$.

Corollaire 4.28. Les fonctions $t \mapsto \phi_{i}(t), i=1,2$ sont $C^{2}$ à gauche et à droite en 0 . 
On a donc, par exemple à gauche :

$$
\ddot{\phi}_{1}\left(0^{-}\right)=\alpha+\beta u_{1}\left(0^{-}\right), \ddot{\phi}_{2}\left(0^{-}\right)=\delta u_{2}\left(0^{-}\right) .
$$

On peut alors poser :

$$
\phi_{1}(t)=\frac{1}{2}\left(\alpha+\beta u_{1}\left(0^{-}\right)\right) t^{2}+\mathrm{o}\left(t^{2}\right), \phi_{2}(t)=\frac{1}{2} \delta u_{2}\left(0^{-}\right) t^{2}+\mathrm{o}\left(t^{2}\right) .
$$

On déduit de l'expression du contrôle extrémal en fonction de $\phi_{1}, \phi_{2}$ :

$$
u_{1}\left(0^{-}\right)=-\frac{\alpha+\beta u_{1}\left(0^{-}\right)}{\sqrt{\left(\alpha+\beta u_{1}\left(0^{-}\right)\right)^{2}+\left(\delta u_{2}\left(0^{-}\right)\right)^{2}}}, u_{2}\left(0^{-}\right)=\frac{-\delta u_{2}\left(0^{-}\right)}{\sqrt{\left(\alpha+\beta u_{1}\left(0^{-}\right)\right)^{2}+\left(\delta u_{2}\left(0^{-}\right)\right)^{2}}} .
$$

On distingue deux cas selon que $u_{2}\left(0^{-}\right)=0$ ou $u_{2}\left(0^{-}\right) \neq 0$.

- Si $u_{2}\left(0^{-}\right)=0$ alors $u_{1}\left(0^{-}\right)=-$signe $\left(\alpha+\beta u_{1}\left(0^{-}\right)\right)= \pm 1$, ce qui impose la contrainte suivante :

- si $u_{1}\left(0^{-}\right)=+1$ on doit avoir $\alpha+\beta<0$;

- si $u_{1}\left(0^{-}\right)=-1$ on doit avoir $\alpha-\beta>0$.

- Si $u_{2}\left(0^{-}\right) \neq 0$ alors nécessairement $\delta \neq 0$, et on obtient, sous réserve que $|\alpha| \leq|\beta+\delta|$ :

$$
u_{1}\left(0^{-}\right)=\frac{-\alpha}{\beta+\delta}, u_{2}\left(0^{-}\right)= \pm \sqrt{1-\left(\frac{\alpha}{\beta+\delta}\right)^{2}} .
$$

Les résultats sont les mêmes pour la limite à droite.

Ainsi :

Lemme 4.29. Sous l'hypothèse $(H)$, en un point de $\Sigma_{2}^{\prime}$ se raccordent au moins deux, et au plus quatre extrémales régulières. La fonction de commutation est de classe $C^{2}$ à gauche et à droite et admet un point de rebroussement au point de commutation.

Remarque 4.30. On peut immerger dans le flot des extrémales précédentes les extrémales correspondant à $u_{2}=0$, qui jouent un rôle particulier dans le problème et sont décrites par le lemme suivant.

Lemme 4.31. Supposons $u_{2}=0$. Alors $\ddot{\phi}_{2}=0$ et $u_{1}= \pm 1$. Au point de commutation d'ordre 2 on a $\ddot{\phi}_{1} \neq 0$ pour $u_{1}=+1$ et $u_{1}=-1$. Alors les extrémales du système mono-entrée $\dot{q}=\bar{X}+u_{1} \bar{Y}_{1}$, où $\chi=k \pi$, représentées sur la figure 12, sont solutions et correspondent à trois situations dites parabolique, hyperbolique ou elliptique.

1. En un point parabolique le contrôle extrémal est donné par $u_{1}=-$ signe $\phi_{1}$ et la fonction de commutation admet un point de rebroussement.

2. En un point hyperbolique il passe trois extrémales : $u=+1, u=-1$ et $u_{s}$ contrôle singulier admissible, et il y a possibilité de connecter ces trois extrémales.

3. En un point elliptique il ne passe qu'une extrémale singulière admissible mais non optimale.

Les connections 1 et 2 sont représentées sur la figure 13.

Démonstration. Les trois cas correspondent à la situation de [14] pour les systèmes mono-entrée. Montrons que l'on peut les immerger comme des extrémales de notre problème. Pour $u_{2}=0, \chi=k \pi$ est invariant et on peut immerger les extrémales du système mono-entrée avec $p_{\chi}=0$, i.e. $\phi_{2}=0$.

\subsubsection{Existence de trajectoires optimales et relaxation}

Il résulte de notre analyse et des travaux de [10] :

Proposition 4.32. Sur un domaine de vol où $\chi \neq k \pi$ il n'existe pas de trajectoire singulière, et les trajectoires extrémales du problème coïncident avec celles du problème relaxé et sont bang-bang avec une borne uniforme 


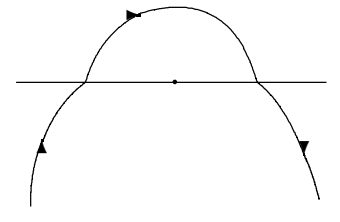

cas parabolique

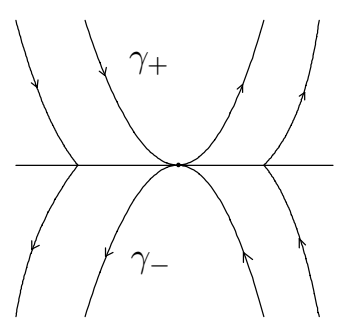

cas hyperbolique

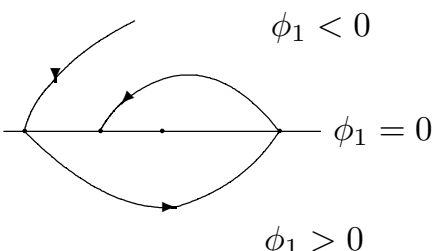

cas elliptique

FIGURE 12

sur le nombre de commutations. Sinon il existe des trajectoires singulières, et si elles sont admissibles pour le problème relaxé alors :

- si elles sont elliptiques, il n'existe pas de borne uniforme sur le nombre de commutations des trajectoires extrémales ;

- si elles sont hyperboliques alors elles sont pour certaines conditions initiales $C^{0}$-optimales si $u_{2}=0$, et donc il n'existe pas de trajectoire optimale car elles doivent être approchées par relaxation.

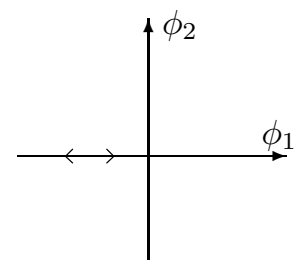

cas parabolique

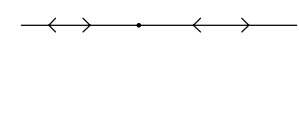

cas hyperbolique

FigURE 13

Conclusion. L'analyse des extrémales régulières est incomplète. Pour compléter l'étude il faut à partir de (43) calculer toutes les trajectoires qui vont ou partent de la surface de commutation. Cela est suffisant pour décrire la politique de commutation. La classification topologique permet de compter les tours. L'analyse de la structure des extrémales doit être complétée par le calcul des extrémales optimales. Le point important est le calcul des points conjugués qui permettent de contrôler le nombre d'oscillations des trajectoires optimales, ce qui généralise le problème de contrôler le nombre de commutations des trajectoires optimales dans le cas monoentrée (voir $[19,20])$. Le concept de point conjugué est identique à celui de la géométrie Riemannienne ou sous-Riemannienne, et est aisément calculable d'un point de vue numérique. Un point intéressant est aussi d'analyser les extrémales lorsque le contrôle est $v=\dot{\mu}$; le système est alors affine en $v$ et toutes les extrémales sont singulières.

\section{Le PROBlème DE CONTRÔle OPTIMAL AVEC LES CONTRAINTES SUR L'ÉtAT}

L'objectif de cette section est d'initialiser l'analyse du problème de contrôle optimal en prenant en compte les contraintes sur l'état. On rappelle un principe du minimum [15] (conditions nécessaires d'optimalité en boucle ouverte) adapté à notre analyse. Notre contribution essentielle est de faire une évaluation de l'ensemble des états accessibles en temps petit, en prenant en compte les contraintes du problème, et en se limitant au sous-système I. Ce travail utilise l'évaluation de l'ensemble des états accessibles dans le cas non contraint de la section 4 , et on construit une forme normale du système contraint. Le concept fondamental est le concept 
d'ordre de la contrainte. On obtient des conditions nécessaires et suffisantes d'optimalité de l'arc frontière, sous des conditions génériques adaptées à notre étude, et la loi optimale au voisinage d'un point frontière est calculée en boucle fermée (synthèse optimale).

\subsection{Le concept d'ordre et l'évaluation de l'ensembles des états accessibles du système contraint}

On considère le système affine mono-entrée :

$$
\dot{q}=X(q)+u Y(q),|u| \leq 1
$$

et le problème de minimiser le temps de transfert sous la contrainte :

$$
c(q) \leq 0
$$

où $X, Y$ et $c$ sont lisses, et les conditions limites sont : $q(0)=q_{0}, q(T)=q_{1}$.

Le problème de minimisation est noté $\left(P_{0}\right)$ et peut être immergé dans la famille à un paramètre de problèmes $\left(P_{\alpha}\right)$ où la contrainte est :

$$
c(q) \leq \alpha, \alpha \text { petit. }
$$

Définition 5.1. L'ordre absolu (ou générique) de la contrainte est l'entier $m$ tel que :

$$
Y . c=Y X . c=\cdots=Y X^{m-2} . c=0 \text { et } Y X^{m-1} . c \neq 0
$$

où les champs de vecteurs $X, Y$ agissent sur $c$ par dérivée de Lie.

Définition 5.2. Un arc frontière $t \mapsto \gamma_{b}(t)$ est une solution non triviale du système entièrement contenue dans $c=0$.

Si l'ordre absolu de la contrainte est $m$, un arc frontière peut être génériquement calculé en dérivant $m$ fois la fonction $t \mapsto c(q(t))$ et en résolvant par rapport à $u$ l'équation linéaire :

$$
c^{(m)}=X^{m} \cdot c+u Y X^{m-1} \cdot c=0 .
$$

Un arc frontière est donc contenu dans :

$$
c=\dot{c}=\cdots=c^{(m-1)}=0 .
$$

La contrainte $c=0$ est dite primaire et les contraintes $\dot{c}=\cdots=c^{(m-1)}=0$ sont dites secondaires.

On note :

$$
u_{b}=-\frac{X^{m} \cdot c}{Y X^{m-1} \cdot c}
$$

le feedback associé à un arc frontière.

Hypothèses C. Soit $t \mapsto \gamma_{b}(t), t \in[0, T]$ un arc frontière. On introduit les hypothèses suivantes :

$\left(C_{1}\right) Y X^{m-1} . c / \gamma \neq 0$ où $m$ est l'ordre de la contrainte.

$\left(C_{2}\right)\left|u_{b}(t)\right|<1$ pour $\left.t \in\right] 0, T$ [, c'est-à-dire que le contrôle frontière est admissible.

$\left(C_{3}\right)\left|u_{b}(t)\right|<1$ pour $t \in[0, T]$, c'est-à-dire que le contrôle frontière est non saturant.

Calcul de l'ordre des contraintes pour le problème de la navette

Flux thermique. La contrainte sur le flux thermique est :

$$
c_{1}=C_{q} \sqrt{\rho} v^{3} \leq \alpha_{1}, \text { où } \rho=\rho_{0} \mathrm{e}^{-\beta r}
$$


et $\dot{c}_{1}=\varphi_{1}(r, v)+\varphi_{2}(r, v) \sin \gamma=0$ est une contrainte secondaire. De plus :

$$
\ddot{c}_{1}=\varphi_{3}(r, v, \gamma)+u \varphi_{4}(r, v) \cos \gamma
$$

avec $\varphi_{4}=-k^{\prime} C_{q} \rho^{3 / 2}\left(3 g v^{3}+\frac{\beta}{2} v^{5}\right) \neq 0$.

Accélération normale. La contrainte est :

$$
c_{2}=\gamma_{n_{0}} \rho v^{2} \leq \alpha_{2}
$$

On obtient :

$$
\dot{c}_{2}=-\gamma_{n_{0}}\left(2 k \rho^{2} v^{3}+\left(\beta \rho v^{3}+2 g \rho v\right) \sin \gamma\right)
$$

et $\dot{c}_{2}=\varphi_{5}(r, v)+\varphi_{6}(r, v) \sin \gamma=0$ est une contrainte secondaire. De plus :

$$
\ddot{c}_{2}=\varphi_{7}(r, v, \gamma)+u \varphi_{8}(r, v) \cos \gamma
$$

avec $\varphi_{8}=-k^{\prime} \gamma_{n_{0}} \rho^{2}\left(\beta v^{4}+2 g v^{2}\right) \neq 0$. On a donc montré :

Lemme 5.3. Pour la navette spatiale, dans le domaine de vol où $\cos \gamma \neq 0$, les contraintes sur le flux thermique et l'accélération normale sont d'ordre 2, et l'hypothèse $\left(C_{1}\right)$ est vérifiée le long d'un arc frontière.

\subsection{Un Principe du Minimum avec contraintes sur l'état [15]}

\subsubsection{Temps fixé}

On considère un système mono-entrée :

$$
\dot{q}=X(q)+u Y(q),|u| \leq 1
$$

et un coût à minimiser de la forme :

$$
J(u)=G(q(T))
$$

où le temps de transfert $T$ est fixé et $q$ vérifie la contrainte :

$$
c(q) \leq 0
$$

Les conditions frontières sont de la forme:

$$
q(0)=q_{0}, \Phi(q(T))=0 .
$$

Soit $t \mapsto \gamma_{b}(t), t \in[0, T]$ un arc frontière. On suppose que les hypothèses $\left(C_{1}\right)$ et $\left(C_{2}\right)$ sont satisfaites.

Conditions nécessaires (Principe du Minimum)

Soit $t \mapsto q(t), t \in[0, T]$ une trajectoire optimale, lisse par morceaux. On introduit le Hamiltonien :

$$
H(q, p, u, \eta)=\langle p, X+u Y\rangle+\eta c
$$

où $\langle$,$\rangle est le produit scalaire, p \neq 0$ est le vecteur adjoint (représenté par un vecteur ligne), et $\eta$ est le multiplicateur de Lagrange de la contrainte. Sous les hypothèses $\left(C_{1}, C_{2}\right)$, satisfaites le long des arcs frontières, 
les conditions nécessaires de [15] sont les suivantes :

1. Il existe $\eta(t) \geq 0$ et des réels $\eta_{0} \geq 0, \sigma$ tels que le vecteur adjoint vérifie:

$$
\begin{gathered}
\dot{p}=-p\left(\frac{\partial X}{\partial q}+u \frac{\partial Y}{\partial q}\right)-\eta \frac{\partial c}{\partial q} \quad \text { p.p. } \\
p(T)=\eta_{0} \frac{\partial \Phi}{\partial q}(q(T))+\sigma \frac{\partial G}{\partial q}(q(T)) .
\end{gathered}
$$

2. La fonction $\eta(t)$ vérifie

$$
\forall t \in[0, T] \quad \eta(t) c(q(t))=0
$$

et est continue à l'intérieur de l'arc frontière.

3. La condition de saut en un temps $t_{1}$ de contact ou de jonction avec la frontière est :

$$
p\left(t_{1}^{+}\right)=p\left(t_{1}^{-}\right)-\nu_{1} \frac{\partial c}{\partial q}\left(q\left(t_{1}\right)\right), \nu_{1} \geq 0 .
$$

4. Le contrôle optimal $u(t)$ minimise presque partout le Hamiltonien :

$$
H(q(t), p(t), u(t), \eta(t))=\min _{|v| \leq 1} H(q(t), p(t), v, \eta(t)) .
$$

\section{Remarque 5.4.}

1. Dans ce principe du minimum, seule la contrainte c est pénalisée. D'autres choix sont possibles en utilisant en particulier les contraintes secondaires, voir [12,18].

2. Il existe un principe du minimum très général, sans aucune hypothèse sur l'ordre de la contrainte, voir par exemple [11], où l'équation adjointe (58) prend la forme:

$$
p(t)=-\int p(s)\left(\frac{\partial X}{\partial q}(q(s))+u \frac{\partial Y}{\partial q}(q(s))\right) \mathrm{d} s-\int \frac{\partial c}{\partial q}(q(s)) \mathrm{d} \mu
$$

où $\mu$ est une mesure dont le support est contenu dans $c=0$. Nos conditions nécessaires sont plus précises car la mesure prend la forme $: \mathrm{d} \mu=\eta(t) \mathrm{d} t$ où $\eta$ est continue. Si la condition $\left(C_{1}\right)$ n'est pas vérifiée, alors en un point non générique où $Y X^{m-1} . c=0, \eta$ peut exploser.

\subsubsection{Le problème du temps minimal}

Le temps de transfert est ici non fixé. On reparamétrise les trajectoires sur $[0,1]$ en procédant ainsi : on pose $s=\frac{t}{T}$ et $z=T$. Le problème est alors de minimiser $t$ pour le système :

$$
\frac{\mathrm{d} q}{\mathrm{~d} s}=(X+u Y) z, \frac{\mathrm{d} t}{\mathrm{~d} s}=z, \frac{\mathrm{d} z}{\mathrm{~d} s}=0 .
$$

Le Hamiltonien s'écrit :

$$
H=\langle p,(X+u Y) z\rangle+p_{t} z+\eta c
$$

et les conditions de transversalité impliquent :

$$
p_{t} \geq 0 \text { en } s=1, p_{z}=0 \text { en } s=0,1 .
$$

Le système adjoint est :

$$
\begin{gathered}
\frac{\mathrm{d} p}{\mathrm{~d} s}=-p\left(\frac{\partial X}{\partial q}+u \frac{\partial Y}{\partial q}\right) z-\eta \frac{\partial c}{\partial q} \\
\frac{\mathrm{d} p_{t}}{\mathrm{~d} s}=0 \text { et } \frac{\mathrm{d} p_{z}}{\mathrm{~d} s}=-p(X+u Y)-p_{t}
\end{gathered}
$$


et en utilisant de plus [11] on a :

$$
M=\max _{|v| \leq 1} H=0
$$

et donc $p_{z}=0$. En paramétrant par $t$ et en changeant $\eta$ en $\frac{\eta}{z}$ et $M$ en $\frac{M}{z}$ on obtient les conditions suivantes.

Conditions nécessaires pour le problème du temps minimal

$$
\begin{aligned}
& \dot{q}=(X+u Y) z \quad \text { p.p. } \\
& \dot{p}=-p\left(\frac{\partial X}{\partial q}+u \frac{\partial Y}{\partial q}\right) z-\eta \frac{\partial c}{\partial q} \quad \text { p.p. } \\
& u\langle p, Y\rangle=\min _{|v| \leq 1} v\langle p, Y\rangle \quad \text { p.p. } \\
&\left(p, p_{t}\right) \neq(0,0) \quad \text { et } \quad M=0 .
\end{aligned}
$$

La condition de saut est :

$$
p\left(t_{1}^{+}\right)=p\left(t_{1}^{-}\right)-\nu_{1} \frac{\partial c}{\partial q}\left(q\left(t_{1}\right)\right), \nu_{1} \geq 0
$$

De plus $p_{t} \geq 0, \eta \geq 0$ avec $\eta=0$ si $c<0$, et $t \mapsto \eta(t)$ est continue le long de la frontière $c=0$.

Définition 5.5. Pour le problème du temps minimal on utilise la terminologie suivante. On appelle extrémale une solution $\left(q, p, \eta, \nu_{i}\right)$ des équations précédentes. Elle est dite exceptionnelle si $p_{t}=0$. Dans le cas non exceptionnel on fait la normalisation : $p_{t}=\frac{1}{2}$. On note $\Phi=\langle p, Y(q)\rangle$ la fonction de commutation et $\Sigma_{s}$ la surface de commutation formée des points où le contrôle optimal est discontinu.

\subsection{Calcul des paramètres $\left(\eta, \nu_{i}\right)$}

Dans le principe du minimum sans contrainte sur l'état, les indéterminées sont le vecteur adjoint et le temps, calculés avec les conditions limites sur l'état. Dans le cas contraint, il y a des indéterminées supplémentaires : $\left(\eta, \nu_{i}\right)$. La contribution essentielle de [15] est de les calculer en utilisant le concept d'ordre. On va présenter ces calculs dans le cas de l'ordre $m=1$ ou 2. Nos formules sont de nature géométrique et utilisent les crochets de Lie itérés de $X, Y$ agissant sur les contraintes.

\subsubsection{Le cas $m=1$}

Lemme 5.6. Supposons que l'ordre est $m=1$. Alors :

1. Le long de l'arc frontière on a:

$$
\eta=\frac{\langle p,[X, Y](q)\rangle}{(Y . c)(q)} .
$$

2. Supposons que le contrôle est discontinu lors du contact ou de la jonction d'un arc bang-bang avec la frontière. Alors $\nu_{1}=0$.

Démonstration. Montrons le premier point. Le long de l'arc frontière, la relation $(61)$ et l'hypothèse $\left(C_{2}\right)$ donnent : $\Phi(t)=\langle p, Y(q(t))\rangle=0$. En dérivant il vient :

$$
\dot{\Phi}=\langle p,[X, Y]\rangle-\eta Y . c=0
$$

et $Y . c \neq 0$ car l'arc est d'ordre 1. On en déduit la relation cherchée. 
Prouvons le deuxième point. On note $a=X . c$ et $b=Y . c$; alors $\dot{c}=a+u b$. Soit $Q$ un point de contact d'un arc extrémal bang-bang $t \mapsto q(t)$ avec la frontière, en un temps $t_{1}$. Soit $\varepsilon>0$ assez petit ; il vient : $c\left(q\left(t_{1}-\varepsilon\right)\right)<0$ et $c\left(q\left(t_{1}+\varepsilon\right)\right)<0$. En passant à la limite $\varepsilon \rightarrow 0$ on obtient :

$$
(a+b u)\left(t_{1}^{-}\right) \geq 0,(a+b u)\left(t_{1}^{+}\right) \leq 0
$$

et en prenant la différence :

$$
b\left(t_{1}\right)\left(u\left(t_{1}^{-}\right)-u\left(t_{1}^{+}\right)\right) \geq 0 .
$$

Supposons par exemple que $b\left(t_{1}\right)>0$. On a alors : $u\left(t_{1}^{-}\right)-u\left(t_{1}^{+}\right) \geq 0$, et $u\left(t_{1}^{-}\right) \neq u\left(t_{1}^{+}\right)$par hypothèse.

D'après le principe du minimum, la fonction de commutation vérifie :

$$
\Phi\left(t_{1}^{-}\right) \leq 0 \text { et } \Phi\left(t_{1}^{+}\right) \geq 0 .
$$

D'après (67) il vient :

$$
\Phi\left(t_{1}^{+}\right)=\Phi\left(t_{1}^{-}\right)-\nu_{1} b\left(t_{1}\right)
$$

On en déduit : $\nu_{1} b\left(t_{1}\right) \leq 0$, et d'après le principe du minimum $\nu_{1} \geq 0$. Si $\nu_{1} \neq 0$ on a $b\left(t_{1}\right) \leq 0$, et cela contredit $b\left(t_{1}\right)>0$. Le cas $b\left(t_{1}\right)<0$ est similaire. Le point 2 est donc prouvé dans le cas d'un point de contact. Le cas d'un point de jonction avec un arc frontière se traite de façon semblable.

5.3.2. Le cas $m=2$

Lemme 5.7. Supposons que l'ordre est $m=2$. Alors :

1. Le long de l'arc frontière on a:

$$
\eta=\frac{\langle p,[X,[X, Y]]\rangle+u_{b}\langle p,[Y,[X, Y]]\rangle}{[X, Y] . c} .
$$

2. En un point de contact ou de jonction : $\Phi\left(t_{1}^{-}\right)=\Phi\left(t_{1}^{+}\right)$.

3. En un point d'entrée :

$$
\nu_{1}=\frac{\dot{\Phi}\left(t_{1}^{-}\right)}{([X, Y] \cdot c)\left(q\left(t_{1}\right)\right)} .
$$

Démonstration. On a $Y . c=0$. Notons $a=X^{2} . c$ et $b=Y X . c$.

Prouvons 1. Le long de la frontière on a $\Phi=\langle p, Y\rangle=0$, et en dérivant il vient :

$$
\begin{aligned}
& \dot{\Phi}=\langle p,[X, Y]\rangle=0 \\
& \ddot{\Phi}=\langle p,[X,[X, Y]]\rangle+u_{b}\langle p,[Y,[X, Y]]\rangle-\eta \frac{\partial c}{\partial q} \cdot[X, Y] .
\end{aligned}
$$

Or $\frac{\partial c}{\partial q} \cdot[X, Y]=[X, Y] . c=(X Y-Y X) . c$, et $Y . c=0$ car $m=2$. Donc :

$$
[X, Y] . c=-Y X . c=-b .
$$

En particulier on en déduit 1.

Prouvons 2. En un point de contact ou de jonction on sait que $\Phi\left(t_{1}^{+}\right)=\Phi\left(t_{1}^{-}\right)-\nu_{1}$ Y.c. Or Y.c $=0$, ce qui donne 2 . 
Prouvons 3. En un point d'entrée on a :

$$
\begin{aligned}
0=\dot{\Phi}\left(t_{1}^{+}\right) & =\left\langle p\left(t_{1}^{+}\right),[X, Y]\left(q\left(t_{1}\right)\right)\right\rangle \\
& =\left\langle p\left(t_{1}^{-}\right),[X, Y]\left(q\left(t_{1}\right)\right)\right\rangle-\nu_{1} \frac{\partial c}{\partial q} \cdot[X, Y] \\
& =\dot{\Phi}\left(t_{1}^{-}\right)-\nu_{1}[X, Y] . c \\
& =\dot{\Phi}\left(t_{1}^{-}\right)+\nu_{1} Y X . c .
\end{aligned}
$$

En particulier on obtient 3 .

\subsection{Synthèses locales temps-minimum pour des systèmes plans avec contraintes sur l'état}

L'objectif de cette section est d'initialiser la classification des synthèses temps-minimales au voisinage de la contrainte, pour les systèmes plans. C'est une étape préliminaire introduisant les outils méthodologiques pour analyser le problème de la navette.

\subsubsection{Généralités}

On considère un système $\dot{q}=X+u Y,|u| \leq 1, q=(x, y) \in \mathbb{R}^{2}$, avec une contrainte $c(q) \leq 0$. On note $\omega=p d q$ la forme horloge définie sur le lieu où $X$ et $Y$ sont indépendants par : $\omega(X)=1$ et $\omega(Y)=0$. Les trajectoires singulières sont localisées sur $S: \operatorname{det}(Y,[X, Y])=0$, et le contrôle singulier $u_{s}$ vérifie : $\langle p,[X,[X, Y]]\rangle+$ $u_{s}\langle p,[Y,[X, Y]]\rangle=0$. La 2-forme $d \omega$ est nulle sur $S$.

Soit $q_{0}$ tel que $c\left(q_{0}\right)=0$, identifié à 0 . Le problème est de déterminer le statut d'optimalité locale d'un arc frontière $t \mapsto \gamma_{b}(t)$, issu de 0 et correspondant au contrôle $u_{b}$, et de décrire la synthèse optimale au voisinage de $q_{0}$. La première étape est de construire une forme normale.

Lemme 5.8. Supposons :

1. $X\left(q_{0}\right), Y\left(q_{0}\right)$ sont linéairement indépendants ;

2. la contrainte est d'ordre 1, c'est-à-dire Y.c $\left(q_{0}\right) \neq 0$.

Alors en changeant si nécessaire $u$ en $-u$, il existe un difféomorphisme local préservant 0 tel que le système contraint s'écrit :

$$
\begin{aligned}
& \dot{x}=1+y a(x, y) \\
& \dot{y}=b(x, y)+u, \quad y \leq 0 .
\end{aligned}
$$

Démonstration. En utilisant un changement local de coordonnées, on peut identifier $Y$ à $\frac{\partial}{\partial y}$ et l'arc contraint à $\gamma_{b}: t \mapsto(t, 0)$. L'espace d'états admissibles est soit $y \leq 0$ soit $y \geq 0$. En changeant éventuellement $u$ en $-u$ on peut l'identifier à $y \leq 0$.

\subsubsection{Le cas générique}

Dans la forme normale précédente on a :

- $Y\left(q_{0}\right),[X, Y]\left(q_{0}\right)$ indépendants $\Leftrightarrow a(0) \neq 0$;

- le contrôle frontière en 0 est admissible et non saturant $\Leftrightarrow|b(0)|<1$.

Modèle local. Analysons la synthèse optimale locale au voisinage de 0 , sous les hypothèses précédentes. On pose $a=a(0)$ et $b=b(0)$. Le modèle local s'écrit :

$$
\begin{aligned}
& \dot{x}=1+a y \\
& \dot{y}=b+u, \quad y \leq 0 .
\end{aligned}
$$

La forme horloge est : $\omega=\frac{\mathrm{d} x}{1+a y}$, et on a : $\mathrm{d} \omega=\frac{a}{(1+a y)^{2}} \mathrm{~d} x \wedge \mathrm{d} y$. 
Synthèse locale. Considérons le cas non contraint. D'après le principe du minimum, chaque trajectoire optimale est bang-bang avec au plus une commutation. Plus précisément on a deux cas. Si $a>0$, alors d $\omega>0$ et chaque trajectoire optimale est de la forme $\gamma_{+} \gamma_{-}$(un arc $\gamma_{-} \gamma_{+}$étant temps-maximal). Si $a<0$, alors d $\omega<0$ et un arc $\gamma_{-} \gamma_{+}$(resp. $\gamma_{+} \gamma_{-}$) est temps-minimal (resp. temps-maximal). En utilisant encore la forme horloge, le même raisonnement montre que l'arc frontière $\gamma_{b}$ est optimal si et seulement si $a>0$. On représente la synthèse optimale pour le problème contraint sur la figure 14 .

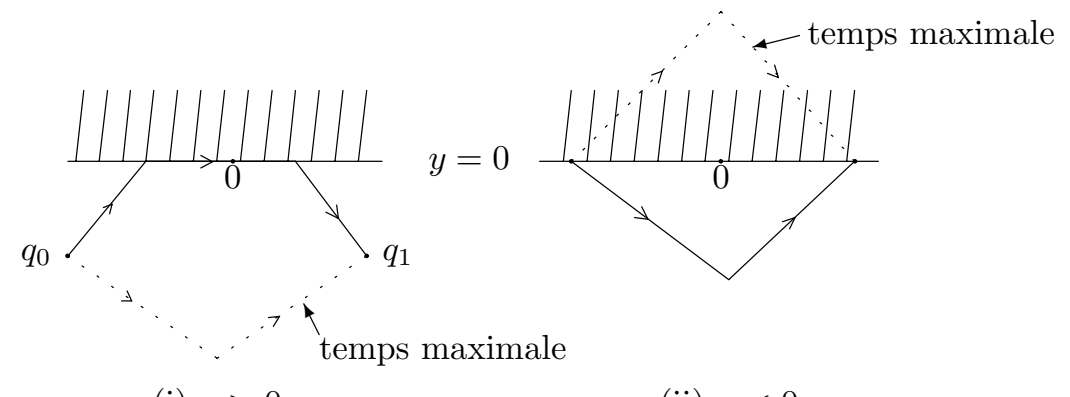

(i) $a>0$

FIGURE 14

\section{Lien avec le principe du minimum}

Le long de la frontière, on a d'après le lemme 5.6 :

$$
\langle p, Y\rangle=0 \text { et } \eta=\frac{\langle p,[X, Y](q)\rangle}{(Y . c)(q)} .
$$

En notant $p=\left(p_{x}, p_{y}\right)$, on obtient $\eta=-a p_{x}$, et $p_{x}$ est orienté par :

$$
\langle p, X+u Y\rangle+p_{t}=0, p_{t} \geq 0
$$

Donc $p_{x}<0$. On en déduit que signe $\eta=$ signe $a$, et la condition nécessaire d'optimalité $\eta>0$ est violée si $a<0$.

On résume nos résultats par :

\section{Lemme 5.9.}

1. Pour le problème non contraint, si $a>0$ un arc $\gamma_{+} \gamma_{-}$est temps minimal et un arc $\gamma_{-} \gamma_{+}$est temps maximal, et inversement si $a<0$.

2. Pour le problème contraint, un arc frontière est optimal si et seulement si $a>0$, et dans ce cas chaque politique optimale est de la forme $\gamma_{+} \gamma_{b} \gamma_{-}$.

\subsubsection{Le cas singulier}

Dans la forme normale du lemme 5.8 , si $Y$ et $[X, Y]$ sont dépendants en 0 , alors $a(0)=0$. Faisons alors l'hypothèse que le lieu $S: \operatorname{det}(Y,[X, Y])=0$ est une courbe simple. Puisque $Y$ est normalisé à $\frac{\partial}{\partial y}$ et l'arc frontière à $\gamma_{b}: t \mapsto(t, 0)$, la pente de $S$ en 0 est un invariant. Dans le modèle local on approche $S$ par une droite et le modèle est :

$$
\begin{aligned}
& \dot{x}=1+y(a y+b x) \\
& \dot{y}=c+u, \quad y \leq 0
\end{aligned}
$$

où $a, b$ sont des constantes. Le lieu $S$ est : $2 a y+b x=0$. D'après [5] l'arc singulier $\gamma_{s}$ pour le système sans 
contrainte, et avec $u \in \mathbb{R}$, est soit $C^{0}$-temps minimal soit maximal, et le test d'optimalité est la condition de Legendre-Clebsch :

- $a<0$ : temps minimal ;

- $a>0$ : temps maximal.

Le contrôle singulier laisse $S$ invariante et vérifie :

$$
b(1+y(a y+b x))+2 a\left(c+u_{s}\right)=0 .
$$

Sa valeur en 0 est : $u_{s}=-c-\frac{b}{2 a}$, avec $a \neq 0$. La condition d'admissibilité du contrôle singulier est : $\left|c+\frac{b}{2 a}\right|<1$. La forme horloge est :

$$
\omega=\frac{\mathrm{d} x}{a y^{2}+b x y} \text {. }
$$

Donc $\mathrm{d} \omega=\frac{2 a y+b x}{a y^{2}+b x y} \mathrm{~d} x \wedge \mathrm{d} y$, et $\mathrm{d} \omega$ s'annule sur $S$. De plus signe $\mathrm{d} \omega=$ signe $(2 a y+b x)$. On va effectuer l'analyse dans le cas suivant :

Cas $\mathbf{a}<\mathbf{0}$ et $\left|\mathbf{c}+\frac{\mathbf{b}}{\mathbf{2} \mathbf{a}}\right|<\mathbf{1}, \mathbf{b} \neq \mathbf{0}$. On a deux situations, selon le signe de $b$. Si $b>0$, l'arc frontière est optimal pour $x \geq 0$ et non optimal pour $x<0$. La synthèse optimale est représentée sur la figure 15 i. Le cas $b<0$ est similaire, et la synthèse est représentée sur la figure 15 ii.

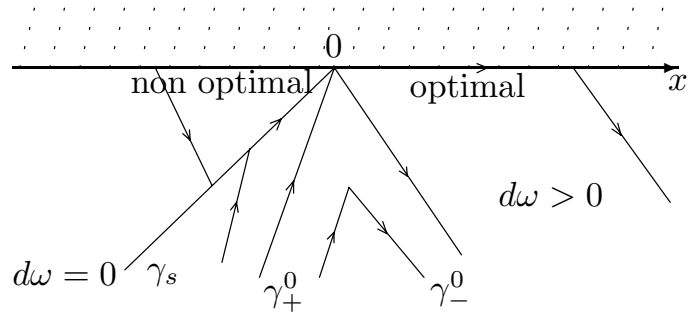

(i) $\operatorname{cas} b>0$

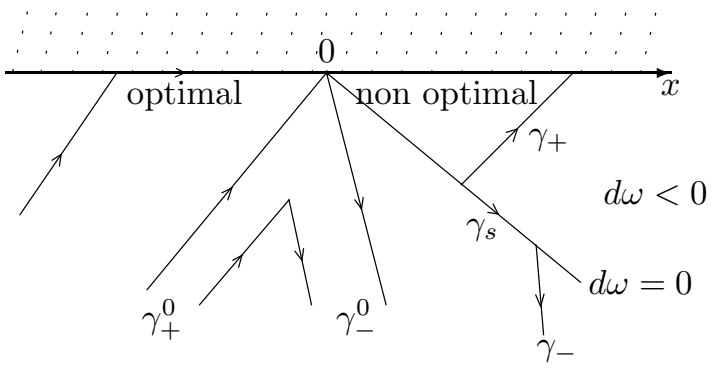

(ii) cas $b<0$

FiguRE 15

Lemme 5.10. Supposons $a<0,\left|c+\frac{b}{2 a}\right|<1$ et $b \neq 0$. Alors chaque trajectoire optimale au voisinage de 0 a au plus trois commutations. De plus :

- si $b>0$, la synthèse optimale locale est représentée sur la figure 15i, et un arc optimal est de la forme $\gamma_{ \pm} \gamma_{s} \gamma_{b} \gamma_{-}$

- si $b<0$, la synthèse optimale locale est représentée sur la figure 15ii, et un arc optimal est de la forme $\gamma_{+} \gamma_{b} \gamma_{s} \gamma_{ \pm}$

\subsection{Synthèse locale temps-minimale pour le sous-système I avec contraintes sur l'état}

On va construire la synthèse optimale locale pour le problème de la navette. La première étape est de construire une forme normale.

\subsubsection{Forme normale au voisinage de la contrainte pour la navette}

Les champs $X, Y$ et $[X, Y]$ sont indépendants, et $[Y,[X, Y]] \in \operatorname{Vect}\{Y,[X, Y]\}$. La contrainte est d'ordre 2 . On suppose que les hypothèses $\left(C_{1}\right)$ et $\left(C_{3}\right)$ sont satisfaites le long de l'arc frontière $\gamma_{b}$, c'est-à-dire que $Y X . c \neq 0$ et que le contrôle $u_{b}$ est admissible et non saturant. L'étude est localisée en $q_{0}=0$. On note $q=(x, y, z)$.

Normalisation 1. Puisque $Y(0) \neq 0$, on identifie localement $Y$ à $\frac{\partial}{\partial z}$. Les difféomorphismes locaux $\varphi=$ $\left(\varphi_{1}, \varphi_{2}, \varphi_{3}\right)$ préservant 0 et $Y$ vérifient alors : $\frac{\partial \varphi_{1}}{\partial z}=\frac{\partial \varphi_{2}}{\partial z}=0$ et $\frac{\partial \varphi_{3}}{\partial z}=1$. Puisque la contrainte est d'ordre 2 , on a $Y . c=0$ au voisinage de 0 et donc $Y$ est tangent à toutes les surfaces $c=\alpha, \alpha$ petit. En particulier : $\frac{\partial c}{\partial z}=0$. 
Normalisation 2. Puisque $c$ ne dépend pas de $z$, en utilisant un difféomorphisme local préservant 0 et $\frac{\partial}{\partial z}$, on peut identifier la contrainte à $c=x$. Le système s'écrit alors :

$$
\dot{x}=X_{1}(q), \dot{y}=X_{2}(q), \dot{z}=X_{3}(q)+u
$$

et $x \leq 0$. On suppose que l'arc frontière est un $\operatorname{arc} \sigma$ dans $x=\dot{x}=0$ passant par 0 . En gardant une approximation affine, suffisante pour notre analyse, on obtient le modèle local suivant :

$$
\begin{aligned}
& \dot{x}=a_{1} x+a_{2} y+a_{3} z \\
& \dot{y}=b_{0}+b_{1} x+b_{2} y+b_{3} z \\
& \dot{z}=c_{0}+c_{1} x+c_{2} y+c_{3} z+u
\end{aligned}
$$

où l'approximation de $\sigma$ est la droite : $x=0, a_{2} y+a_{3} z=0$.

Normalisation 3. On se restreint au plan $x=0$. En utilisant une transformation de la forme $Z=\alpha y+z$, on peut normaliser l'arc frontière $\sigma$ à : $x=z=0$. En changeant $y$ en $\lambda y$, l'arc frontière peut être identifié à $t \mapsto(0, t, 0)$. En changeant si nécessaire $z$ en $-z$ et $u$ en $-u$, on peut de plus supposer $a_{3}>0$. On a montré :

Lemme 5.11. Sous nos hypothèses, le modèle local est :

$$
\begin{aligned}
& \dot{x}=a_{1} x+a_{3} z \\
& \dot{y}=1+b_{1} x+b_{2} y+b_{3} z \\
& \dot{z}=c+u+c_{1} x+c_{2} y+c_{3} z, \quad|u| \leq 1
\end{aligned}
$$

où $a_{3}>0$ et la contrainte est $x \leq 0$. L'arc frontière est identifié à $\sigma: t \mapsto(0, t, 0)$. Puisqu'il est admissible et non saturant en 0 , on a $|c|<1$. De plus $a_{3}=-[X, Y]$.c.

Théorème 5.1. Considérons le problème du temps minimal pour le système $\dot{q}=X(q)+u Y(q), q \in \mathbb{R}^{3}$, avec la contrainte $c(q) \leq 0$. Soit $q_{0} \in\{c=0\}$. Faisons les hypothèses suivantes :

1. au voisinage de $q_{0}:[Y,[X, Y]] \in \operatorname{Vect}\{Y,[X, Y]\}$;

2. $X, Y,[X, Y]$ sont linéairement indépendants en $q_{0}$, et $[X,[X, Y]]\left(q_{0}\right)=a X\left(q_{0}\right)+b Y\left(q_{0}\right)+c[X, Y]\left(q_{0}\right)$ avec $a<0$;

3. la contrainte est d'ordre 2 et les hypothèses $\left(C_{1}\right)$ et $\left(C_{3}\right)$ sont satisfaites en $q_{0}$.

Alors l'arc frontière issu de $q_{0}$ est localement temps-minimal si et seulement si la trajectoire $\gamma_{-}\left(q_{0}\right)$ est contenue dans le domaine $c \geq 0$. Dans ce cas la synthèse optimale locale est de la forme $\gamma_{-} \gamma_{+} \gamma_{b} \gamma_{+} \gamma_{-}$.

Démonstration. D'après le lemme 4.14, tout point accessible à partir de $q_{0}$ en temps petit l'est par un arc $\gamma_{+} \gamma_{-} \gamma_{+}$et un arc $\gamma_{-} \gamma_{+} \gamma_{-}$, et d'après le théorème 4.1 , pour le problème non contraint une politique $\gamma_{-} \gamma_{+} \gamma_{-}$ est temps-minimale et une politique $\gamma_{+} \gamma_{-} \gamma_{+}$est temps-maximale.

Considérons le modèle construit précédemment où $q_{0}=0$ et l'arc frontière $\gamma_{b}$ est identifié à $t \mapsto(0, t, 0)$. Soit $B=\gamma_{b}(t), t>0$ petit. Pour une trajectoire issue de $\left(0, y_{0}, 0\right)$ et associée à $u=+1$ ou -1 , on a les approximations :

$$
\begin{aligned}
& x(t)=\frac{a_{3}}{2}\left(c_{0}+y_{0}+u\right) t^{2}+\mathrm{o}\left(t^{2}\right) \\
& z(t)=\left(c_{0}+y_{0}+u\right) t+\mathrm{o}(t)
\end{aligned}
$$

et les projections dans le plan $(x, z)$ des arcs $\gamma_{+} \gamma_{-} \gamma_{+}$et $\gamma_{-} \gamma_{+} \gamma_{-}$joignant 0 à $B$ sont des boucles $\tilde{\gamma}_{+} \tilde{\gamma}_{-} \tilde{\gamma}_{+}$et $\tilde{\gamma}_{-} \tilde{\gamma}_{+} \tilde{\gamma}_{-}$, représentées sur la figure 16 .

La boucle $\tilde{\gamma}_{-} \tilde{\gamma}_{+} \tilde{\gamma}_{-}\left(\right.$resp. $\left.\tilde{\gamma}_{+} \tilde{\gamma}_{-} \tilde{\gamma}_{+}\right)$est contenue dans $x \leq 0$ (resp. $x \geq 0$ ). 


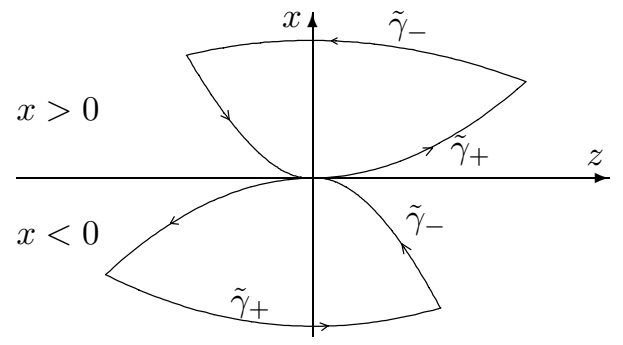

FIGURE 16

Dans les coordonnées initiales, si l'arc $\gamma_{-} \gamma_{+} \gamma_{-}$joignant 0 à $B$ est contenu dans $c \leq 0$, alors il est tempsminimal et l'arc frontière n'est pas optimal. S'il est contenu dans $c \geq 0$, on peut joindre 0 à $B$ par un $\operatorname{arc}$ $\gamma_{+} \gamma_{-} \gamma_{+}$contenu dans $c \leq 0$, temps-maximal. Dans ce cas clairement l'arc frontière $\gamma_{b}$ est temps-minimal.

La synthèse optimale est alors la suivante. On peut utiliser le modèle local. Soient $B_{1}<0<B_{2}$ deux points de la frontière. Considérons les arcs $\gamma_{-} \gamma_{+}$arrivant en $B_{1}$ et les arcs $\gamma_{+} \gamma_{-}$partant de $B_{2}$, voir figure 17. En faisant varier $B_{1}$ et $B_{2}$ on décrit entièrement l'ensemble des trajectoires optimales.

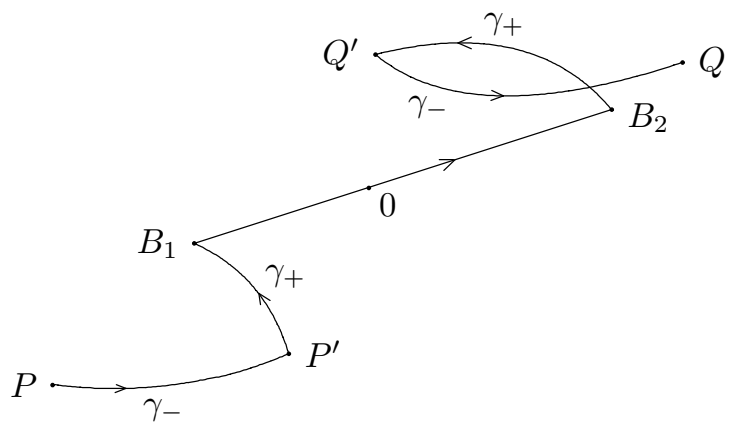

FIGURE 17

Remarque 5.12. On peut aisément calculer la politique optimale joignant $P$ à $Q$ en utilisant le modèle : $\dot{x}=a_{3} z, \dot{y}=1, \dot{z}=c+u+y$.

\subsubsection{Lien avec le principe du minimum}

D'après le lemme 5.7 , on a :

$$
\eta=\frac{\langle p,[X,[X, Y]]\rangle}{[X, Y] \cdot c}
$$

et $[X,[X, Y]]=a X+b Y+c[X, Y]$, avec $\langle p, Y\rangle=\langle p,[X, Y]\rangle=0$, le long de la frontière. Donc $\eta=\frac{a\langle p, X\rangle}{[X, Y] \cdot c}$. D'après le principe du minimum on a $\langle p, X\rangle<0$, et dans le modèle : $[X, Y] \cdot c=-a_{3}<0$. La condition nécessaire $\eta \geq 0$ donne $a \geq 0$. Dans ce cas $\gamma_{+} \gamma_{-} \gamma_{+}$est la politique optimale pour le problème non contraint, et les boucles correspondantes de la figure 16 sont contenues dans le domaine $x \leq 0$. La condition nécessaire d'optimalité $\eta \geq 0$ est violée si et seulement si $a<0$ dans la forme normale et le principe du minimum détecte l'optimalité de l'arc frontière.

Considérons la politique optimale pour joindre $P$ à $Q$, représentée sur la figure 17 . Les sauts $\nu_{1}$ et $\nu_{2}$ du vecteur adjoint au point d'entrée $B_{1}$ et au point de sortie $B_{2}$ avec la frontière sont donnés par les arcs extrémaux du problème non contraint joignant respectivement $P$ à $B_{1}$ et $B_{2}$ à $Q$. Sur ces arcs le vecteur adjoint est entièrement déterminé par la condition de commutation en $B_{1}, P^{\prime}$ et $B_{2}, Q^{\prime}$ (voir Fig. 17). 
En un point de contact avec la frontière, l'arc $\gamma_{-} \gamma_{+} \gamma_{-}$est une solution optimale du problème non contraint et vérifie le principe du minimum avec $\nu_{1}=0$, mais aussi pour une famille à un paramètre $\nu_{1} \in[0, \bar{\nu}], c f$. [15].

\subsubsection{Application à la navette}

Dans notre problème on a $a<0$. On doit situer les boucles associées à $\gamma_{-} \gamma_{+} \gamma_{-}$où $\gamma_{-}$correspond à $\cos \mu=-1$, par rapport au domaine admissible. D'après les calculs de la section 5.1 on a pour les contraintes $c_{1}$ et $c_{2}$ :

$$
\ddot{c}_{i}=\Phi(r, v, \gamma)+u \cos \gamma \bar{\Phi}(r, v)
$$

où $\bar{\Phi}<0$. Donc $\ddot{c}_{i}$ est minimal lorsque $\mu=0$, soit $u=+1$. Supposons donc que les paramètres du problème sont tels que l'hypothèse $\left(C_{3}\right):\left|u_{b}\right|<1$ le long de l'arc frontière soit vérifiée. Alors les arcs $\gamma_{-}$issus d'un point frontière $c=0 \mathrm{du}$ domaine admissible sont contenus dans le domaine non admissible et l'arc frontière est optimal.

\section{Simulations numÉRIQUeS}

Dans cette section on simule les solutions du principe du minimum de Maurer appliqué au sous-système (I) de la navette, voir figure 18. On utilise le logiciel MATLAB et un intégrateur permettant de trouver les racines de fonctions associées à la solution du système, ce qui permet de gérer les contraintes.
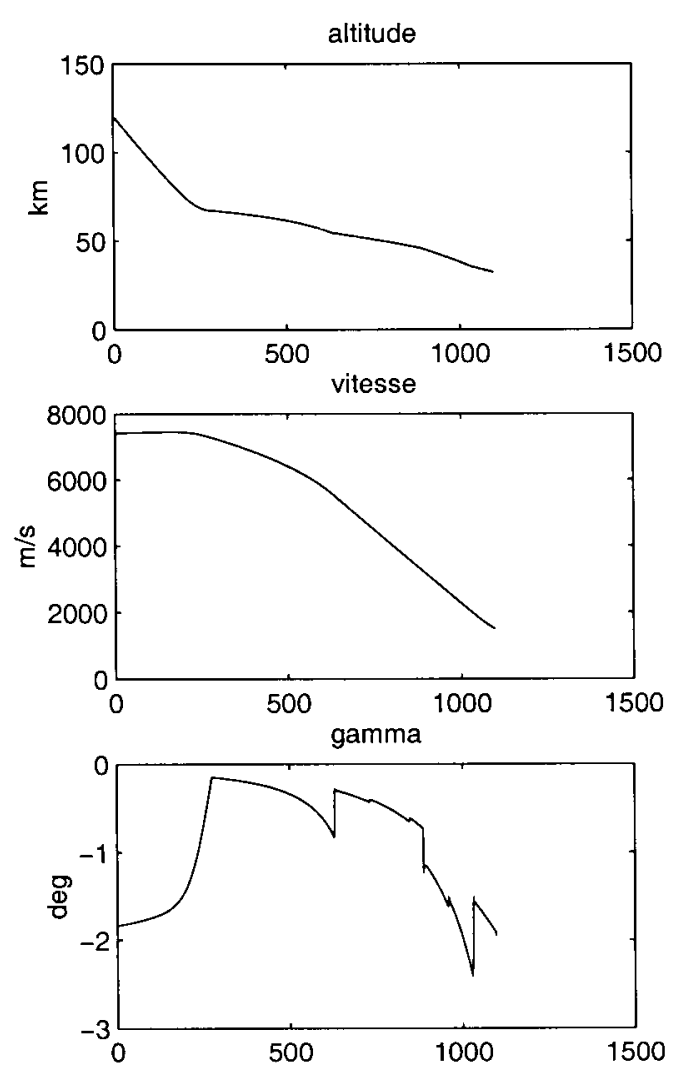
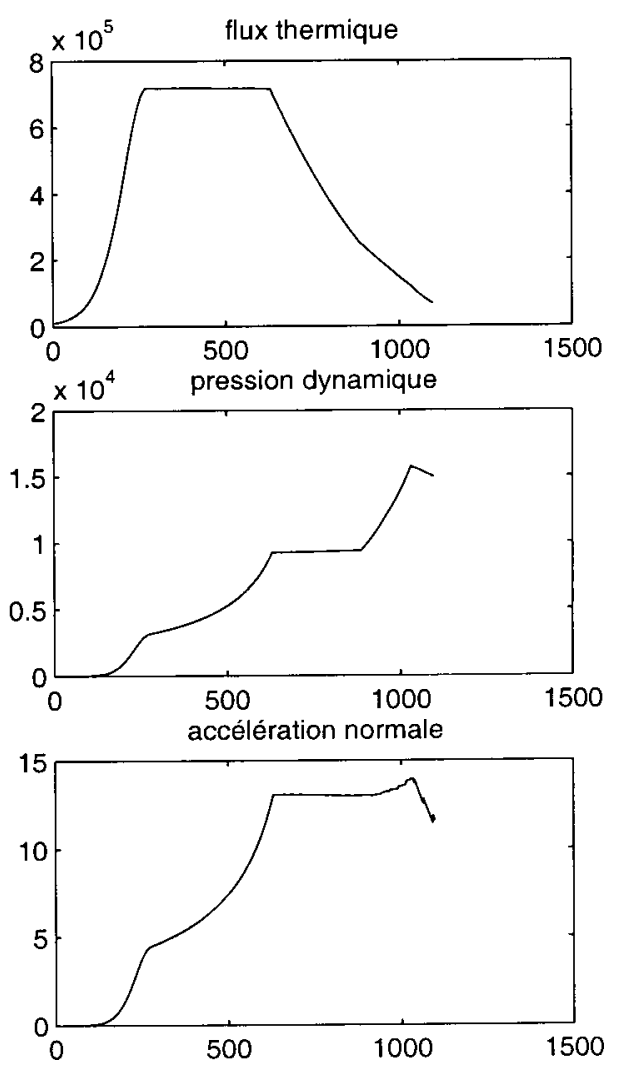

FIGURE 18 
Le modèle de la densité atmosphérique et des coefficients $C_{D}, C_{L}$ est donné par le cahier des charges du CNES. On a effectué les simulations numériques pour différentes valeurs du vecteur adjoint, et obtenu, à la main, une trajectoire ayant pour conditions aux limites :

- Conditions initiales :

$$
r_{0}=6497960 \mathrm{~m}, v_{0}=7404,95 \mathrm{~m} / \mathrm{s}, \gamma_{0}=-1,84 \mathrm{deg} .
$$

- Conditions finales :

$$
r_{1}=6410300 \mathrm{~m}, v_{1}=1470 \mathrm{~m} / \mathrm{s}, \gamma_{1}=-2,12 \mathrm{deg} .
$$

Partant du point initial, on suit un morceau d'arc non contraint, au cours duquel on a une commutation (le contrôle commute de +1 à -1 ), puis heurte la contrainte sur le flux thermique. On suit alors un arc frontière (phase dite iso-flux), puis un arc saturant la contrainte sur l'accélération normale. Enfin on rejoint le point final. Notons que la contrainte sur la pression dynamique n'est pas active.

Des simulations plus précises utilisant une méthode de type multiple-shooting (voir $[16,17]$ ) doivent être conduites. Cette étude préliminaire permet cependant de deviner une bonne condition initiale sur le vecteur adjoint. En effet ces méthodes numériques sont basées sur des algorithmes récursifs permettant d'améliorer une estimation des conditions initiales sur le vecteur adjoint, de manière à satisfaire la condition finale sur l'état. La principale difficulté de ces méthodes est de trouver une bonne estimation du vecteur adjoint initial, qui produise une solution proche des condition terminales souhaitées. Elle est due au fait que les extrémales sont très sensibles à des petites perturbations des conditions aux limites (en particulier à cause de la forme Hamiltonienne des équations).

\section{Conclusion}

L'objectif de ce travail a été de placer le problème de contrôle optimal dans un contexte géométrique et d'initialiser l'étude des différents problèmes à analyser pour le résoudre.

L'approche géométrique consiste à caractériser la politique optimale locale en termes de crochets de Lie formés à partir des champs de vecteurs décrivant le système et agissant par dérivée de Lie sur la contrainte.

Dans le cas non contraint, cet objectif est rempli pour le sous-système I décrivant l'évolution des variables $(r, v, \gamma)$. Pour le système complet nos résultats sont partiels. Par ailleurs on a choisi de normaliser la cible au pôle nord, ce qui a conditionné nos calculs et a créé une singularité au point terminal. Un problème non trivial à résoudre est de compléter l'analyse des extrémales régulières du problème convexifié. Il est lié à la construction des modèles locaux pour approcher le système. Enfin on doit caractériser les trajectoires optimales, ce qui pose en particulier le problème de calcul des point conjugués.

Pour le problème de contrôle optimal avec contraintes sur l'état, on a introduit la méthodologie pour la classifier la synthèse optimale locale, et on a comparé cette classification avec les conditions nécessaires du principe du minimum. On a montré que l'arc frontière est en général optimal pour le problème de la navette.

La classification locale des synthèses optimales est le point crucial pour l'étude globale en utilisant des simulations numériques. On peut prévoir qu'un problème optimal avec contraintes du niveau de complexité du problème de rentrée atmosphérique est résoluble en complétant notre analyse géométrique par des simulations numériques fondées sur le principe du minimum.

\section{RÉFÉRENCES}

[1] H. Baumann et H.J. Oberle, Numerical computation of optimal trajectories for coplanar aeroassisted orbital transfer. J. Optim. Theory Appl. 107 (2000) 457-479.

[2] O. Bolza, Calculus of variations. Chelsea (1973).

[3] F. Bonnans et G. Launay, Large scale direct optimal control applied to the re-entry problem. J. Guidance, Control and Dynamics 21 (1998) 996-1000.

[4] B. Bonnard et G. Launay, Time minimal control of batch reactors. ESAIM: COCV 3 (1998) 407-467. 
[5] B. Bonnard et I. Kupka, Théorie des singularités de l'application entrée/sortie et optimalité des singulières. Forum Math. 5 (1993) 111-159.

[6] A. Bryson et Y.C. Ho, Applied optimal control. Hemisphere Pub. Corporation (1975).

[7] J.B. Caillau et J. Noailles, Coplanar control of a satellite around the Earth. ESAIM: COCV 6 (2001) 239-258.

[8] CNES, Mécanique spatiale. Cepadues Eds. (1993).

[9] J.M. Coron et L. Praly, Guidage en rentrée atmosphérique, Rapport 415. CNES (2000).

[10] I. Ekeland, Discontinuité des champs de vecteurs extrémaux en calcul des variations. Publ. Math. IHES 47 (1977) 5-32.

[11] A.D. Ioffe et V.M. Tikhomirov, Theory of extremal problems. North Holland (1979).

[12] P.H. Jacobson et al., New necessary conditions of optimality for control problems with state-variable inequality constraints. J. Math. Anal. 35 (1971) 255-284.

[13] A.J. Krener et H. Schättler, The structure of small time reachable sets in small dimensions. SIAM J. Control Optim. 27 (1989) $120-147$.

[14] I. Kupka, Geometric theory of extremals in optimal control problems. Trans. Amer. Math. Soc. 299 (1987) $225-243$.

[15] H. Maurer, On optimal control problems with bounded state variables and control appearing linearly. SIAM J. Control Optim. 15 (1977) 345-362.

[16] A. Miele, Recent advances in the optimization and guidance of aeroassisted orbital transfers. Acta Astronautica 38 (1996) 747-768.

[17] H.J. Pesch, A practical guide to the solution of real-life optimal control problems. Control Cybernet. 23 (1994).

[18] V. Pontryagin et al., Méthodes mathématiques des processus optimaux. Eds. Mir (1974).

[19] H. Schättler, The local structure of time-optimal trajectories in dimension 3 under generic conditions. SIAM J. Control Optim. 26 (1988) 899-918.

[20] H.J. Sussmann, The structure of time-optimal trajectories for single-input systems in the plane: The $C^{\infty}$ non singular case. SIAM J. Control Optim. 25 (1987) 856-905. 